\title{
Focal Sleep Spindle Deficits Reveal Focal Thalamocortical Dysfunction and Predict Cognitive Deficits in Sleep Activated Developmental Epilepsy
}

\author{
Mark A. Kramer, ${ }^{1}$ Sally M. Stoyell, ${ }^{2}$ Dhinakaran Chinappen, ${ }^{2}$ Lauren M. Ostrowski, ${ }^{2}$ Elizabeth R. Spencer, ${ }^{1}$ \\ Amy K. Morgan, ${ }^{3}$ Britt Carlson Emerton, ${ }^{3}$ Jin Jing, ${ }^{2,4}$ M. Brandon Westover, ${ }^{2,4}$ Uri T. Eden, ${ }^{1}$ Robert Stickgold, ${ }^{4,5}$ \\ ${ }^{\mathbb{D}}$ Dara S. Manoach, ${ }^{3,4,6}$ and ${ }^{\circledR}$ Catherine $\mathrm{J}$. $\mathrm{Chu}^{2,5}$ \\ ${ }^{1}$ Department of Mathematics and Statistics, Boston University, Boston, Massachusetts $02215,{ }^{2}$ Department of Neurology, Massachusetts General \\ Hospital, Boston, Massachusetts 02114, ${ }^{3}$ Department of Psychiatry, Massachusetts General Hospital, Boston, Massachusetts 02114, ${ }^{4}$ Harvard \\ Medical School, Boston, Massachusetts 02115, ${ }^{5}$ Department of Psychiatry, Beth Israel Deaconess Medical Center, Boston, Massachusetts 02215, and \\ ${ }^{6}$ Athinoula A. Martinos Center for Biomedical Imaging, Charlestown, Massachusetts 02129
}

Childhood epilepsy with centrotemporal spikes (CECTS) is the most common focal epilepsy syndrome, yet the cause of this disease remains unknown. Now recognized as a mild epileptic encephalopathy, children exhibit sleep-activated focal epileptiform discharges and cognitive difficulties during the active phase of the disease. The association between the abnormal electrophysiology and sleep suggests disruption to thalamocortical circuits. Thalamocortical circuit dysfunction resulting in pathologic epileptiform activity could hinder the production of sleep spindles, a brain rhythm essential for memory processes. Despite this pathophysiologic connection, the relationship between spindles and cognitive symptoms in epileptic encephalopathies has not been previously evaluated. A significant challenge limiting such work has been the poor performance of available automated spindle detection methods in the setting of sharp activities, such as epileptic spikes. Here, we validate a robust new method to accurately measure sleep spindles in patients with epilepsy. We then apply this detector to a prospective cohort of male and female children with CECTS with combined high-density EEGs during sleep and cognitive testing at varying time points of disease. We show that: (1) children have a transient, focal deficit in spindles during the symptomatic phase of disease; (2) spindle rate anticorrelates with spike rate; and (3) spindle rate, but not spike rate, predicts performance on cognitive tasks. These findings demonstrate focal thalamocortical circuit dysfunction and provide a pathophysiological explanation for the shared seizures and cognitive symptoms in CECTS. Further, this work identifies sleep spindles as a potential treatment target of cognitive dysfunction in this common epileptic encephalopathy.

Key words: automated spindle detection; BECTS; CECTS; latent state model; Rolandic epilepsy

Significance Statement

Childhood epilepsy with centrotemporal spikes is the most common idiopathic focal epilepsy syndrome, characterized by selflimited focal seizures and cognitive symptoms. Here, we provide the first evidence that focal thalamocortical circuit dysfunction underlies the shared seizures and cognitive dysfunction observed. In doing so, we identify sleep spindles as a mechanistic biomarker, and potential treatment target, of cognitive dysfunction in this common developmental epilepsy and provide a novel method to reliably quantify spindles in brain recordings from patients with epilepsy.

Received July 31, 2020; revised 0ct. 16, 2020; accepted Nov. 16, 2020.

Author contributions: M.A.K., S.M.S., D.C., L.M.O., A.K.M., B.C.E., and C.J.C. performed research; M.A.K., D.C., E.R.S., U.T.E., and C.J.C. analyzed data; M.A.K. and C.J.C. wrote the first draft of the paper; M.A.K., S.M.S., D.C., L.M.O., E.R.S., A.K.M., B.C.E., R.S., D.S.M., and C.J.C. edited the paper; M.A.K. and C.J.C. wrote the paper; J.J., M.B.W., R.S., and D.S.M. contributed unpublished reagents/analytic tools; C.J.C. designed research.

M.A.K. was supported by National Science Foundation DMS Award 1451384. E.R.S. was supported by National Science Foundation GRFP. C.J.C. was supported by National Institutes of Health K23 Award NS092923. C.J.C., M.A.K., and D.S.M. were supported by National Institutes of Health National Institute of Neurological Disorders and Stroke R01NS115868.

The authors declare no competing financial interests.

Correspondence should be addressed to Catherine J. Chu at cjchu@mgh.harvard.edu.

https://doi.org/10.1523/JNEUROSCI.2009-20.2020

Copyright $\odot 2021$ the authors

\section{Introduction}

Childhood epilepsy with centrotemporal spikes (CECTS, previously benign epilepsy with centrotemporal spikes) is the most common idiopathic focal epilepsy, accounting for $\sim 10 \%$ of all childhood onset epilepsies (Astradsson et al., 1998; Larsson and Eeg-Olofsson, 2006; Callenbach et al., 2010; Camfield and Camfield, 2014; Berg and Rychlik, 2015). The epilepsy in CECTS is characterized by seizures and spikes arising independently from the left and right centrotemporal regions during non-rapid eye movement (NREM) sleep (Bouma et al., 1997; Callenbach et al., 2010; Carvill et al., 2013; Tsai et al., 2013; Ross et al., 2020). 
Clinically, CECTS is now recognized as an epileptic encephalopathy, belonging to a group of developmental disorders characterized by abundant sleep-activated spikes that emerge alongside new cognitive deficits (Berg et al., 2010; Carvill et al., 2013). In CECTS, the most common difficulties are noted in general intellectual functioning, fine motor coordination, processing speed, and speech-sound processing (Vannest et al., 2015; Scheffer et al., 2017; Wickens et al., 2017). Among these concerns, the largest deficits relate to consolidation of new information (Scheffer et al., 2017). Both the epilepsy and cognitive symptoms spontaneously resolve in CECTS across a range of ages, but always by late adolescence (Ross et al., 2020).

Despite detailed clinical and electrophysiological observations of CECTS, the cause of this common epilepsy is unknown. The association between the abnormal electrophysiology and NREM sleep suggests disruption to thalamocortical circuits (McCormick and Bal, 1997; Gent et al., 2018), a pathophysiology historically implicated in idiopathic generalized (Williams, 1953; Beenhakker and Huguenard, 2009), but not focal epilepsies. Sleep spindles, brief bursts of oscillatory $\sigma$ band $(9-15 \mathrm{~Hz})$ activity during NREM sleep, are generated by well-characterized thalamocortical circuits (Beenhakker and Huguenard, 2009). Similar to the epileptiform spikes in CECTS, sleep spindles can be focal and are most prominent in the Rolandic cortex (Andersen et al., 1967; Pinto et al., 2014). Prior work has observed that the thalamocortical circuits that generate spindles can be hijacked to support epileptiform spikes (Steriade, 2005; Beenhakker and Huguenard, 2009; Clemente-Perez et al., 2017). If disruptions to thalamocortical circuits underlie the pathologic spikes in CECTS, these processes may also interfere with the generation of healthy spindles.

Efforts to link spike rate with cognitive dysfunction in CECTS and related epileptic encephalopathies have shown inconsistent results (Bjørnæs et al., 2013; Sánchez Fernández et al., 2013; Vega et al., 2018). In contrast, sleep spindles are a well-established mechanism of memory consolidation (Siapas and Wilson, 1998; Walker et al., 2002; Sirota et al., 2003; Rosanova and Ulrich, 2005; Fogel and Smith, 2006; Nishida and Walker, 2007; Peters et al., 2008; Tamaki et al., 2008; Rasch et al., 2009), mediating the synaptic plasticity required for LTP during NREM sleep (Siapas and Wilson, 1998; Sirota et al., 2003; Rosanova and Ulrich, 2005; Beenhakker and Huguenard, 2009). Spindle rate has also been found to correlate with general cognitive performance in children (Wilhelm et al., 2012; Farmer et al., 2018; Hahn et al., 2019).

Despite the shared thalamocortical circuitry with spikes and the functional relevance to normal cognitive processes, the relationship between spindles and the cognitive symptoms in CECTS has not been investigated. We hypothesized that: (1) children with active, but not resolved, CECTS would have a focal disruption in spindle rate in the centrotemporal regions; (2) spindle rate would be inversely related to spike rate; and (3) spindle rate would correlate with neuropsychological performance. Evidence of a spindle disruption in CECTS would demonstrate thalamocortical circuit dysfunction in this idiopathic focal epilepsy and provide a mechanistic explanation for the shared seizures and cognitive symptoms observed.

Although many automated detectors have been developed to quantify spindle activity (Warby et al., 2014), application of these methods to EEG recordings from patients with epilepsy is limited because of the impact of epileptiform spikes on detector performance. Therefore, to test these hypotheses, we introduce and validate a novel automated approach to accurately detect spindles in the setting of epileptiform spikes. We then quantify spindles from NREM EEG recordings in children with CECTS at different stages of the disease and in control subjects, and compare these results to performance on neuropsychological tasks. We provide evidence of a spindle disruption in CECTS, demonstrating thalamocortical circuit dysfunction in this idiopathic focal epilepsy and providing a mechanistic explanation for the shared seizures and cognitive symptoms observed.

\section{Materials and Methods}

Experimental design. All children 4-15 years of age who received a clinical diagnosis of CECTS by a child neurologist following 1989 International League Against Epilepsy criteria (Dreifuss et al., 1989), and confirmed to have a history of focal motor or generalized seizures and an EEG showing sleep activated centrotemporal spikes, were eligible for this prospective study (Fisher et al., 2014). Healthy control school-aged subjects without a history of seizure or known neurologic disorder were also recruited. CECTS and healthy control subjects with a history of unrelated neurologic disease were excluded, although children with attention disorders and mild learning difficulties consistent with known CECTS comorbidities were included (Wickens et al., 2017). Thirty-eight coordinated EEG and neuropsychological visits from 35 subjects (median of $20 \mathrm{~d}$ between EEG recording and neuropsychological test, range 0-111 d) were collected. Twenty-four visits were from children diagnosed with CECTS (mean age 11.4 years, range: 4.9-14.9 years, 17 males), and 14 visits were from control subjects (mean age 12.6 years, range: 8.7-15.1 years, 7 males). Children with CECTS were grouped into two categories of seizure risk: CECTS with active disease (A-CECTS, defined as having had a seizure within the last 12 months, $n=11$ ), and CECTS with resolved disease (R-CECTS, defined as seizure-free for at least 12 months, $n=13$ ). We chose these definitions because the majority of children with CECTS who are seizure-free for 1 year (including both treated and untreated) have a sustained remission (Ross et al., 2020). Three children with CECTS who were initially enrolled during active disease returned after disease resolution (age 9.6, 12, 16.8 years; 1 male). Twelve children (6 subjects with A-CECTS, 6 subjects with RCECTS) were treated with anticonvulsant medication at the time of enrollment.

This research was approved by the Massachusetts General Hospital and Boston University institutional review boards, and assent and informed consent were obtained from each subject and guardian. This experiment was not preregistered.

EEG acquisition and preparation. All subjects arrived to EEG recording sessions after instructions for sleep restriction (recommended maximum of $4 \mathrm{~h}$ of sleep) the prior night, in accordance with our standard clinical protocol. EEG were recorded with a 70 channel cap based on the 10-10 electrode placement system at a $2035 \mathrm{~Hz}$ sampling rate (Easycap, Vectorview, Elekta-Neuromag) with additional electrodes placed at T1 and T2 locations. Subjects were given a nap opportunity lasting up to a maximum of $90 \mathrm{~min}$, between $\sim 9: 00 \mathrm{AM}$ and 11:30 AM, depending on the duration of time required for subject setup and electrode application. EEG data were visually inspected in both an average and a nasal-referential montage by a board-certified neurophysiologist (C.J.C.), and epochs and electrodes without artifact were selected for analysis. All available Stages 2 and 3 NREM sleep epochs were identified for each subject using standard criteria (Grigg-Damberger et al., 2007) and included in analysis (range 77-3592 s, mean $1273 \mathrm{~s}$; no difference in duration of data between CECTS and control groups, two-tailed $t$ test, $p=0.56$ ). Stages 2 and 3 NREM sleep was not scored or analyzed separately. Rapid eye movement (REM) sleep was not observed in any subject. To optimize identification of focal events, all high-density EEG data were rereferenced to the common average reference for subsequent analyses (Nunez and Srinivasan, 2005).

Neuropsychological assessment. Comprehensive neuropsychological assessments were performed on each subject by licensed clinical neuropsychologists (B.C.E., A.K.M., board-certified). To reduce multiple comparisons, we limited our analyses to four measures that capture each of the canonical challenges reported in CECTS: general intellectual functioning, processing speed, fine motor coordination and speed, and 
speech-sound processing (Vannest et al., 2015; Scheffer et al., 2017; Wickens et al., 2017), detailed below.

The Wechsler Intelligence Scale for Children, fifth edition was used to quantify full-scale IQ (FSIQ), an estimate of general intelligence in children derived from subtests of verbal comprehension, perceptual reasoning, working memory, and processing speed (Wechsler, 2014).

Fine motor coordination and speed were assessed using the Grooved Pegboard Task (GPT). This task times the placement of grooved pegs into irregular holes, requiring that the pegs be rotated into the correct position to be successfully placed, thereby providing an assessment of hand-eye coordination, motor speed, and sensorimotor control and integration (Merker and Podell, 2011).

The processing speed index (PSI) from the Wechsler Intelligence Scale for Children, fifth edition was considered separately as a measure for attentional deficiency, as it examines the state of preparedness to respond to stimuli, incorporating both sensory registration and timing of motor response (Jacobson et al., 2011). The PSI subtests require children to attend to visual material and sort or classify targets and symbols in a time-limited setting.

Phonological awareness was assessed using the Comprehensive Test of Phonological Processing, second edition through three composite subscales: the ability to divide a spoken word into its individual phonological components, the ability to blend individual phonemes presented auditorily and articulate them into spoken words, and the ability to break a real word into phonemic pieces, remove one, and combine the remaining pieces together to produce a real word (Wagner et al., 1991).

For all tests, $z$ scores representing each individual's relative deviation from standardized score distributions for their age group, as well as their gender group for the GPT, were computed per task.

Manual spindle detection. We performed manual spindle marking for 18 subjects ( 6 subjects with A-CECTS, 6 subjects with R-CECTS, and 6 control subjects). For each subject, we visually inspected $100 \mathrm{~s}$ of data recorded from 18 channels within the standard 10-20 EEG montage. The start and end of each spindle at each channel was marked by a board certified pediatric neurophysiologist (C.J.C.) following standard criteria (Grigg-Damberger et al., 2007) ( $n=1631$ total manually detected spindles).

Existing automated spindle detector and calculation of $\sigma$ activity. To evaluate performance of standard approaches to detect spindles in patients with epilepsy, we applied an existing spindle detector (Wamsley et al., 2012) to EEG recordings with and without epileptiform activity. We chose this method from the many available automated spindle detectors for three reasons: (1) successful application in existing studies (Wamsley et al., 2012; Warby et al., 2014); (2) balanced performance between sensitivity and positive predictive value (PPV), as shown in a large-scale study (Warby et al., 2014); and (3) reliance on the magnitude of $\sigma$ activity, as this reflects the most common approach implemented in existing detectors (Warby et al., 2014). To compute the magnitude of $\sigma$ activity, we followed the procedure implemented in this detector (Wamsley et al., 2012). First, we applied the continuous Morlet wavelet, squared the (complex) result, and isolated the real part. Then, we computed the absolute value and smoothed the result (moving average $100 \mathrm{~ms}$ ). The spindle detection threshold is set as the median value of the smoothed result, multiplied by an amplification factor; we examined factors between 2 and 12. To demonstrate the impact of spikes on the spindle detection threshold, we compared the average median wavelet value at six electrodes (C3, C5, T3, C4, C6, T4) to the average spike rate from the left and right hemispheres (see Automatic spike detection).

Latent state (LS) spindle detector. Interictal spikes produce wideband spectral features (Kramer et al., 2008) that impact $\sigma$ power and subsequent spindle detections using existing methods. For accurate detection of spindles in the setting of interictal spikes, we developed a new LS spindle detector. We chose this approach for three reasons. First, LS models allow characterization of variables hidden in noisy observed data by leveraging both data features and a dynamical model of the hidden variables that includes history. Here, we model the probability of a spindle given the observed EEG recordings. Second, by modeling the probability of a spindle, the LS model provides an easily interpretable value to determine whether a spindle occurs, or not. Third, the LS modeling framework allows us to explicitly include features computed from the EEG data to mitigate the impact of nonspindle $\sigma$ band activity. Here, we selected three features that separate spindles from both background and spike activity: theta band power, $\sigma$ band power, and a measure of oscillation cycle regularity. As we show in Results, a spindle produces high $\sigma$ band power and oscillation cycle regularity, whereas a spike produces high theta band power, high $\sigma$ band power, and low oscillation cycle regularity. Application of the detector consisted of two steps: (1) training and validation, and (2) implementation on patient and control data.

We designed the LS detector to compute three features. For each EEG channel, we selected a $0.5 \mathrm{~s}$ interval of data and computed the following: (1) the theta power $(4-8 \mathrm{~Hz}),(2)$ the $\sigma$ power $(9-15 \mathrm{~Hz})$, and (3) the Fano factor (here the consistency of time intervals between subsequent peaks and subsequent troughs in the signal). We chose a $0.5 \mathrm{~s}$ interval to balance time and frequency resolution. We expect larger intervals would miss detections of short duration spindles, which are typically required to last at least $0.5 \mathrm{~s}$ (Iber et al., 2007; Purcell et al., 2017; Niethard et al., 2018; Helfrich et al., 2019). The choice of 0.5 s permits a $2 \mathrm{~Hz}$ frequency resolution, compatible with estimating theta band activity $(6 \mathrm{~Hz}$ center frequency, bandwidth $4-8 \mathrm{~Hz})$. A smaller interval would improve the detector's temporal resolution (e.g., support detection of smaller duration spindles), but the impact on frequency resolution would not enable reliable analysis of the theta band. We advanced the $0.5 \mathrm{~s}$ interval by $0.1 \mathrm{~s}$ (overlap $0.4 \mathrm{~s}$ ); the method therefore detected spindles of minimum duration $0.5 \mathrm{~s}$ and with temporal resolution $0.1 \mathrm{~s}$. For (1) and (2), we detrended the data, applied a Hanning taper, computed the power spectrum, and then divided the power at each frequency by the summed total power. Like the automated spindle detector in Wamsley et al. (2012), we computed $\sigma$ power from the (unfiltered) EEG data. For (3), we found the peaks and troughs (minimum peak distance $28 \mathrm{~ms}$, minimum peak prominence $2 \mu \mathrm{V}$ ) of the signal bandpass filtered between $3 \mathrm{~Hz}$ and $25 \mathrm{~Hz}$ (FIR, stop band attenuation $40 \mathrm{~dB}$ at $3 \mathrm{~Hz}$, stop band attenuation $20 \mathrm{~dB}$ at $25 \mathrm{~Hz}$, passband ripple $0.1 \mathrm{~dB}$ ). We used the filtered signal to reduce the variability in peak/trough detections because of higher-frequency activity. We note that the bandpass filtered data were only used when computing the Fano factor; we used the unfiltered EEG data to compute the theta and $\sigma$ power. We computed the time between subsequent peaks, the time between subsequent troughs, and from these interpeak times computed the Fano factor (Eden and Kramer, 2010). We took the natural logarithm of each feature, shifted the interval by $0.1 \mathrm{~s}$, and repeated these steps for the entire duration of the signal.

We note that here we included $\sigma$ activity spanning the $9-15 \mathrm{~Hz}$ band. We chose this frequency interval to broadly accept spindle activity across the wide frequency range observed in the pediatric age group evaluated here (Purcell et al., 2017). We note that an increase in $\sigma$ band power during NREM sleep, reflecting spindle activity, occurred within this frequency range for the subjects considered here.

To train the LS detector, we used the manual spindle detections described in Manual spindle detection. For each channel ( $n=18$ channels per subject) and each subject $(n=18)$, we computed for each $0.5 \mathrm{~s}$ interval ( $0.1 \mathrm{~s}$ shift) the three features and assigned a spindle state label (inspindle or out-spindle) determined from the manual markings. An interval was designated in-spindle when the entire interval lied within the bounds of a manually marked spindle; otherwise, the interval was marked out-spindle. We considered this choice the most conservative option for training the detector; only features extracted from time points completely within a manually marked spindle were designated in-spindle states. We expect that less conservative choices (e.g., at least $50 \%$ of an interval must lie within the bounds of a manually marked spindle) would introduce additional noise; the detector would then be trained to detect spindles using spectral features both inside and outside of manually marked spindles. We therefore required a strict criterion for training that used only features during manually marked spindles. From these data, we fit empirical (Gaussian) likelihood functions to each feature and state, and estimated the transition matrix between the in-spindle and out-spindle states.

The LS detector computes the probability of the in-spindle state in each $0.5 \mathrm{~s}$ interval. To do so, the probabilities of the in-spindle $\left(p_{i n}\right)$ and 
out-spindle $\left(p_{\text {out }}\right)$ states are first initialized at 0.5 . Then, for the first $0.5 \mathrm{~s}$ interval, the transition matrix is applied to $\left[p_{\text {in }}, p_{\text {out }}\right]$ to compute the onestep prediction $\left[p_{\text {in }}^{1}, p_{\text {out }}^{1}\right]$. The three features (power $9-15 \mathrm{~Hz}$, power 4 $8 \mathrm{~Hz}$, interpeak Fano factor) for this interval are then computed, and the likelihood of each feature is used to compute the posterior $\left[p_{i n}^{*}, p_{\text {out }}^{*}\right]$. The posterior is then normalized so $p_{i n}^{*}+p_{\text {out }}^{*}=1$. The value of $p_{i n}^{*}$ is the probability of the in-spindle state for this interval. The interval is shifted forward in time by $0.1 \mathrm{~s}$, and the $\left[p_{\text {in }}, p_{\text {out }}\right]$ for this interval is set to the normalized posterior of the previous interval. This process is repeated for the entire duration of the signal such that each $0.5 \mathrm{~s}$ interval is scored with a probability of being in a spindle state.

To validate the LS detector, we performed a leave-one-out cross validation. To do so, we first trained the LS detector with one subject left out (i.e., with 17 of 18 subjects) on the manually marked data. We then applied the LS detector to compute $p_{\text {in }}$ for each marked electrode of the left-out subject. In this way, we applied the LS detector to data not used to train the LS detector. We repeated this process for all 18 subjects. To identify an automated spindle detection, we must choose a threshold value of $p_{\text {in }}$ above which we declare a spindle occurs. To do so, we evaluated LS detector performance compared with the manual marking using standard measures (see Statistical analysis). We found that a threshold value of 0.95 is optimal. A spindle begins when $p_{\text {in }}$ exceeds the threshold value, and ends when $p_{\text {in }}$ falls below the threshold value. Spindle detections with duration $<0.5 \mathrm{~s}$ were excluded, based on standard requirements (Iber et al., 2007; Purcell et al., 2017; Niethard et al., 2018; Helfrich et al., 2019), and spindles detections separated by $<1 \mathrm{~s}$ were concatenated.

To implement the LS detector on subject data, we applied the LS detector to each electrode from each subject for the entire duration of NREM sleep recording available. We report a spindle detection when $p_{i n}>0.95$ (i.e., when the probability of a spindle is $95 \%$ ).

To analyze narrow frequency intervals corresponding to slow (9$12 \mathrm{~Hz})$ and fast $(12-15 \mathrm{~Hz})$ spindles, we included an additional feature: the instantaneous frequency, computed as the reciprocal of the average interpeak times. Thus, for each interval, we computed four features (power $9-15 \mathrm{~Hz}$, power $4-8 \mathrm{~Hz}$, interpeak Fano factor, and instantaneous frequency), and evaluated the likelihood of each feature during in-spindle and out-spindle states. We set the likelihood of the instantaneous frequency to 1 if the instantaneous frequency lies within the narrow frequency interval of interest (either $9-12 \mathrm{~Hz}$ or 12 $15 \mathrm{~Hz}$ ), and 0 otherwise.

Analysis of spindle features. To assess spindle characteristics, we computed three features: duration, frequency, and amplitude. Duration was defined as the time from the beginning to end of a spindle detection. Frequency was defined as the instantaneous frequency, computed as described in Latent state (LS) spindle detector. Amplitude was defined as the maximum difference between adjacent local maxima and minima (peaks) within the $3-25 \mathrm{~Hz}$ filtered spindle event, computed as described in Latent state (LS) spindle detector. For each patient, we computed the three features for all spindles detected at the centrotemporal channels. We then averaged these features across all detected spindles for a subject. To test for differences in spindle features between groups, we implemented a linear model of the feature with categorical predictors of group (A-CECTS or R-CECTS). We checked for normality of each feature using the Lillietest. For the spindle amplitude and frequency, we found no evidence to reject the null hypothesis that the features come from a normal distribution ( $p=0.14$ for amplitude, and $p=0.21$ for frequency). For spindle duration, we found evidence rejecting the hypothesis that the data come from a normal distribution $(p=0.0089)$. We determined this rejection was because of a single outlier of large duration. Removing this outlier, we found no evidence to reject the null hypothesis $(p=0.38)$. We then found similar qualitative results whether we included or excluded this outlier.

Spectral analysis. To estimate the average power spectrum at the centrotemporal channels for a subject, we first computed the multitaper spectrogram $(T=10 \mathrm{~s}, \mathrm{~W}=1 \mathrm{~Hz}, 19$ tapers, and no overlap between windows) of each channel. We then averaged these spectrogram results over all time and all centrotemporal channels for each subject. From the resulting average power spectrum at the centrotemporal channels for a subject, we determined the peak power in the $\sigma$ band $(9-15 \mathrm{~Hz})$ by applying the function findpeaks.m in MATLAB (minimum peak prominence 1.5 decibels).

Automatic spike detection. For spike detection, we applied two automated spike detection methods: the Persyst 13 spike detector (Persyst Development) and the SpikeNet detector (Jing et al., 2020a,b). We chose to use two automated spike-detection methods for three reasons: (1) manual spike detection approaches have poor inter-rater agreement (Scheuer et al., 2017; Webber et al., 1993); (2) both automated spike detection methods have been validated against manual markings by multiple users (Joshi et al., 2018; Jing et al., 2020a); and (3) as no gold standard exists for automated spike detection, the application of two separate approaches can identify common patterns. We applied both algorithms to the same patients, intervals of data, and electrodes analyzed with the LS spindle detector. While spikes have a broad field and are not localized to a single channel (e.g., see Kramer et al., 2019), the spike detection algorithm in Persyst 13 assigns each spike to a single channel. To prevent incorrect omission of spikes because of channel assignment, we computed spike rate by summing spikes detected at both primary centrotemporal channels and all secondary high-density EEG channels surrounding this ROI (see Fig. 5). Repeating all analyses, including only those spikes detected using Persyst 13 with maximal amplitude in the centrotemporal channels in either hemisphere (C3, C5, T3, and C4, T4, C6), we find qualitatively consistent results. For the SpikeNet detector, which only accepts low-density 10-20 EEG inputs, we computed the spike rate by summing spikes detected with maximal probability in the centrotemporal regions (e.g., C3, T3 and C4, T4).

Statistical analysis. To assess the performance of the two spindle detectors compared with gold standard manual detections and each other, we report PPV, sensitivity, and the F1 score, which is the harmonic mean of the PPV and sensitivity, as follows:

$$
F 1=2 \frac{P P V \times \text { sensitivity }}{P P V+\text { sensitivity }} .
$$

For all measures of detector performance, we chose the threshold with optimum F1 score. We note that, to compute the F1 score, we performed a by-sample analysis, which penalizes detections not perfectly aligned with hand-marked events (Warby et al., 2014). We did so to provide the most rigorous assessment of detector performance and avoid choosing an arbitrary assessment parameter (the overlap threshold) (Warby et al., 2014).

To mitigate the impact of false-positive results following from the multiple testing problem, we tested three a priori hypotheses: (1) that the mean spindle rate is decreased in the centrotemporal regions in children with active CECTS, but not resolved CECTS, compared with controls; (2) that spindle rate is inversely related to spike rate; and (3) that spindle rate in the centrotemporal regions correlates with performance in four neuropsychological tasks.

For hypothesis (1), we modeled the spindle rate as a function of subject group (A-CECTS or R-CECTS) using a generalized linear mixedeffects model (binomial distribution, logit link, and estimated dispersion parameter). For each patient, we computed the average spindle rate in centrotemporal channels (e.g., where spikes are prominent in CECTS) (Xie et al., 2018; Ross et al., 2020) in each hemisphere (left hemisphere: electrodes C3, C5, T3; right hemisphere: C4, C6, T4). We included a random-effects intercept for each patient to account for two separate hemispheric measures from each subject (Gelman, 2005; Winter, 2013).

For hypothesis (2), we modeled the spike rate in the left and right centrotemporal regions as a function of spindle rate in the left and right centrotemporal regions (respectively) using a generalized linear mixedeffects model (binomial distribution, logit link, estimated dispersion parameter, and random-effects intercept for each patient). In this model, the spike rate $s$ is as follows:

$$
s=\frac{e^{\beta_{0}+\beta_{1} d}}{1+e^{\beta_{0}+\beta_{1} d}}
$$

where $d$ is the inverse hyperbolic sine (IHS) transformation of the spindle rate (see next section) and $\beta_{0}, \beta_{1}$ are model parameters to estimate. 
As an approximation of the relationship between spike and spindle rate, we also report $e^{\beta_{1} d}$ for $d$ corresponding to a unit increase in spindle rate.

For hypothesis (3), we modeled the neuropsychological scores as a function of the average spindle rate in the centrotemporal regions. For FSIQ, PSI, and phonological awareness, we first averaged the spindle densities from the left and right centrotemporal channels. Then, to reduce the influence of extreme observations, we applied the IHS transformation to these average spindle densities (Burbidge et al., 1988). We note that the IHS transformation is similar to the logarithmic transformation, but suitable for nonpositive observations. We modeled each neuropsychological score as a function of the IHS transformed spindle densities (linear regression). We note that, for the (single) observation of neuropsychological score per subject, a mixedeffects model with two predictors per subject (i.e., left and right spindle rates) would fail. We therefore averaged the left and right spindle rates to create a single predictor per subject. An alternative approach would be to model the (two) left and right spindle rates per subject with (single) predictor neuropsychological score. In this case, a mixed-effects model would be appropriate. However, we considered this approach less informative; we are interested in predicting the neuropsychological score for a given spindle rate, not vice versa. For GPT, we modeled motor task performance using the right and left hand as a function of the spindle rate in the left and right centrotemporal channels, respectively (linear mixed-effects model, with random-effects intercept for each patient). Because not all subjects performed each neuropsychological assessment, the number of subjects analyzed for each assessment varied as follows: FSIQ ( $n=26,8$ subjects A-CECTS, 8 subjects R-CECTS, 10 control subjects), GPT ( $n=26,7$ subjects A-CECTS, 9 subjects R-CECTS, 10 control subjects), PSI ( $n=28,9$ subjects A-CECTS, 9 subjects R-CECTS, 10 controls), and phonological awareness ( $n=23,6$ subjects A-CECTS, 7 subjects R-CECTS, 10 controls).

As spindle rate may increase across the subject age range included here (Purcell et al., 2017), for each model for each hypothesis, we tested age as a covariate and included age as an independent variable in the model if $p<0.1$. Doing so, we only found evidence to include age in the model of motor task performance (GPT) as a function of spindle rate.

In addition to these four primary hypotheses we conducted exploratory analyses to examine the spatial extent of the spindle deficit, spindle characteristics (duration, frequency, and amplitude), and narrow-band spindle results focused on slow $(9-12 \mathrm{~Hz})$ and fast $(12-15 \mathrm{~Hz})$ spindles (Mölle et al., 2011). We also modeled the neuropsychological scores for the CECTS subjects as functions of the spindle rate, and as functions of the spike rate computed with each of the two spike detection methods. The number of subjects for each assessment matched those listed for CECTS subjects above, except that one A-CECTS subject was omitted from the spike rate computed using Persyst; for this subject, the amount of data available ( $\sim 5 \mathrm{~min})$ was insufficient for detector application.

To correct for multiple comparisons within each hypothesis, we implemented a procedure to control the false detection rate (Benjamini and Hochberg, 1995) with $q=0.05$. The exact $p$ values, effect sizes (i.e., the regression coefficient), and 95\% CIs are reported.

Data accessibility. Raw data were generated at Massachusetts General Hospital and the Athinoula A. Martinos Center for Biomedical Imaging. Derived data are available from the corresponding author on request. Deidentified data may be available on request to the corresponding author with appropriate institutional review board approval.

Software accessibility. Software for the LS spindle detector is available for reuse and further development at https:/github.com/Mark-Kramer/ Spindle-Detector-Method.

\section{Results}

Automated detection of spindles in the setting of spikes

Available automated detectors rely primarily on measures of $\sigma$ activity to detect spindles (example in Fig. 1A) (Warby et al., 2014). However, sharp voltage fluctuations, such as the interictal epileptiform spikes in patients with epilepsy, cause an artifactual increase in $\sigma$ power, resulting in both: (1) false elevations in background $\sigma$ activity, a measure often applied to determine the threshold used to detect spindles; and (2) false detections of these sharp events as spindles (example in Fig. 1B). Notably, even healthy sleep physiology with sharp morphology (e.g., vertex waves or the initial negative deflection in a K-complex) can have this same detrimental effect on spindle detection using available approaches (example in Fig. 1C). To demonstrate the impact of interictal spikes on $\sigma$ band activity, we considered an established spindle detection method that performs well on EEG data from subjects without epilepsy (Wamsley et al., 2012; Warby et al., 2014). We first computed the relationship between spike rate (spikes/min) and median wavelet power in the $\sigma$ band, which determines the spindle detection threshold in this example automated detector (Wamsley et al., 2012). Using two independent automated methods to compute spike rate (see Automatic spike detection), we found a positive relationship between spike rate and median wavelet power in the $\sigma$ band (Persyst: $p=0.00037$, linear regression with predictor logarithm spike rate and outcome median $\sigma$ band wavelet power, $n=23$ patients with CECTS, regression coefficient 1.2e-11, SE 2.8e-12; Fig. 1D; SpikeNet: $p=0.011$, regression coefficient $4.7 \mathrm{e}-12$, SE 1.7e-12). Thus, using approaches that rely primarily on the magnitude of $\sigma$ activity, the presence of spikes results in both falsely detected spindles and an elevated threshold for spindle detection.

To mitigate this concern, we developed a new spindle detector designed to identify spindles in the presence of sharp events (Fig. 2). The proposed LS spindle detector estimates the probability of a spindle given three features calculated from the EEG: (1) the power $9-15 \mathrm{~Hz},(2)$ the power $4-8 \mathrm{~Hz}$, and (3) the Fano factor, which reflects the sinusoidal regularity of rhythmic activity (see Latent state (LS) spindle detector). Using expert markings to train the LS detector (1631 spindles from 18 subjects), we found that intervals with spindles tend to exhibit: (1) high 9$15 \mathrm{~Hz}$ power, (2) low 4-8 $\mathrm{Hz}$ power, and (3) consistent rhythmic activity (Fig. 2A). In comparison, sharp events, such as spikes, are expected to exhibit: (1) high power $9-15 \mathrm{~Hz}$, (2) high power 
A
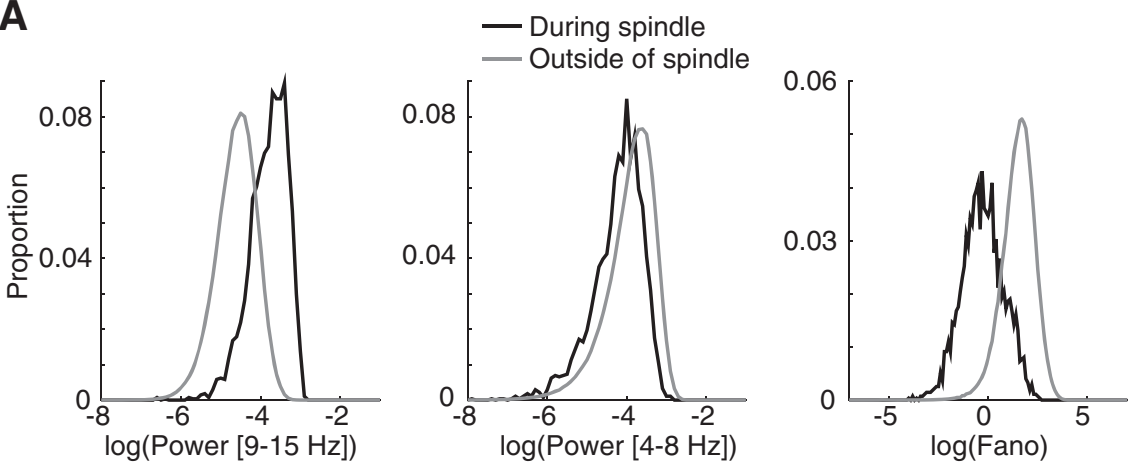

B

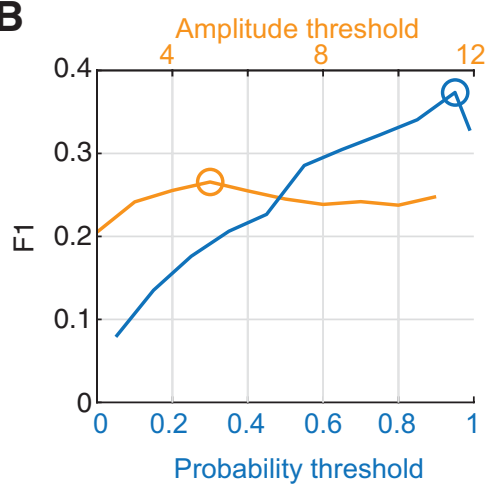

C

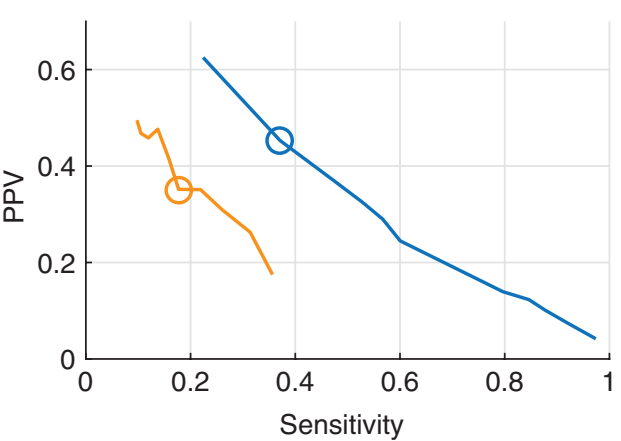

D

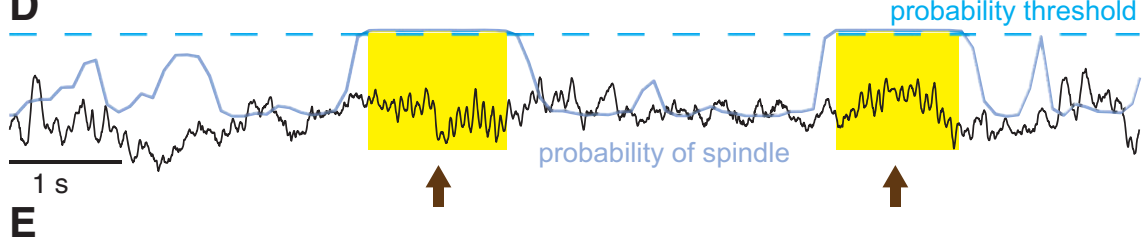

$\underline{E}$
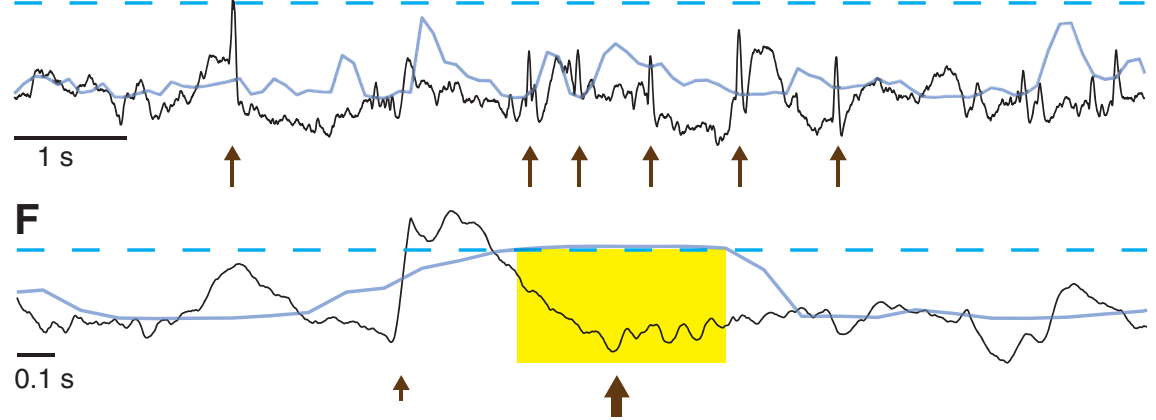

Figure 2. The LS detector outperforms an existing method in the context of epilepsy. $A$, Distributions of the three features used by the $L S$ detector, calculated for the training data. $\boldsymbol{B}, C$, Comparison of the $L S$ detector (blue) and an existing spindle detection method (orange). The LS detector performs with higher $(\boldsymbol{B}) \mathrm{F1}$, (C) sensitivity, and PPV than an existing spindle detector. $\boldsymbol{D}-\boldsymbol{F}$, Illustration of the LS detector (blue curves) applied to example EEG (black) as in Figure 1. When the LS detector exceeds the threshold (dashed line), a spindle is detected. Yellow represents example detections. Spindles $(\boldsymbol{D}, \boldsymbol{F})$ are accurately detected (arrows). Abrupt increases in the EEG because of epileptiform spikes ( $\boldsymbol{E}$, thin arrows) or a K-complex ( $\boldsymbol{F}$, short arrow) are not detected as spindles.

Table 1. Comparative spindle detector performance ${ }^{a}$

\begin{tabular}{llll}
\hline Method & F1 score & PPV & Sensitivity \\
\hline LS detector & 0.37 & 0.45 & 0.37 \\
Existing detector & 0.27 & 0.35 & 0.22 \\
\hline
\end{tabular}

${ }^{\mathrm{a}}$ The $\mathrm{L} S$ detector performs with higher $\mathrm{F} 1$, positive predictive values, and sensitivity than an existing spindle detector.

$4-8 \mathrm{~Hz}$, and (3) irregular rhythmic activity. Upon testing against expert markings, we found that the LS detector identified spindles in epilepsy and control subjects with good performance (F1 score 0.37 ) and outperformed an established spindle detection method (Fig. 2B-F; Table 1). We note that the F1 scores reported here lie within the range of F1 scores computed using a by-sample analysis for existing automated detectors (0.2-0.43) (Warby et al., 2014, their Supplementary Table 1). Therefore, in what follows, we use the LS detector to identify spindles from CECTS and control subjects.

Active, but not resolved, CECTS have a spindle deficit in centrotemporal channels

To assess the relationship between spindle rate (spindles/min) in the affected brain regions and disease state, we first separated the subjects into three groups: (1) those with high seizure risk (active CECTS, A-CECTS), (2) low seizure risk (resolved CECTS, R-CECTS), and (3) controls. We then built a statistical model to relate group to spindle rate in the centrotemporal EEG channels (see Subject recruitment, Statistical analysis). We found that children with A-CECTS have a reduced spindle rate in the centrotemporal regions (estimated rate $1.26 / \mathrm{min}, 95 \% \mathrm{CI}$ $[0.82,1.91] / \mathrm{min})$ compared with control subjects (estimated rate $2.4 / \mathrm{min}$, 95\% CI [1.7, 3.4]/min; effect size 0.51, $95 \%$ CI $[0.29,0.89], p=0.019$; Fig. 3 ). In contrast, we found no evidence that children with R-CECTS have altered spindle rate compared with control subjects (estimated rate 2.2/ min, 95\% CI $[1.5,3.1] / \mathrm{min}, p=0.89)$. We conclude that children with active CECTS have a spindle deficit in the centrotemporal regions. We further conclude that this spindle deficit is transient, present only in CECTS patients with active disease and no longer evident in patients with resolved disease.

\section{CECTS spindle deficit is specific to the centrotemporal regions}

We hypothesized that the spindle deficit in CECTS would be focal and correspond to the centrotemporal cortical regions affected by sleep-activated spikes. To explore the spatial extent of the spindle deficit, we analyzed the spindle rate in six brain regions (Fig. 4A) in each of the three subject groups, based on EEG channel designations (central, temporal, frontal, parietal, occipital, and midline). We found that children with A-CECTS have a reduced spindle rate in the central electrode brain regions compared with controls (factor of $0.55,95 \%$ CI [0.33, 0.93], $p=0.026$; Fig. $4 B$ ). We also found a trend of reduced spindle rate in the temporal regions in A-CECTS compared with controls (factor of $0.53,95 \%$ CI $[0.28,1.02], p=0.057$ ). We did not detect a significant effect of subject group in the other four regions 
( $p>0.22$ for all other regions). We conclude that the spindle deficit observed in patients with active CECTS is focal, localized to the same brain regions affected by epileptiform spikes.

\section{CECTS spindles are inversely correlated with spike rate} Given a proposed shared thalamocortical circuitry underlying NREM sleep-activated spikes and spindles in CECTS, we expect that a patient with more frequent spikes will have less frequent spindles. To test this relationship, we compared the spike and spindle rates for each patient with CECTS (Fig. 5). Using two independent spike detection methods, we found that increased spindle rate anticorrelates with spike rate (Persyst detections: effect size $\beta_{1}=-1.03$, CI $[-1.84,-0.22]$, $p=0.015$; SpikeNet detections: effect size $\beta_{1}=-1.82$, CI $[-2.96,-0.68], p=0.0026$; see Statistical analysis). Thus, a unit increase in spindle rate correlated with a decrease in spike rate by a factor of $\sim 0.2-0.4$. We note that 6 of 11 subjects with active disease, and 5 of 13 subjects with resolved disease, received antiepileptic medication. For both groups, we found no evidence that medication status correlated with spike rate using either detector ( $p>0.25$ in all cases). We found a possible relationship between medication status and spindle rate in subjects with active disease $(p=0.079)$, where subjects on antiepileptic medication had a trend toward a decreased spindle rate. This result may reflect a selection bias in that the subjects with more severe disease were more likely treated with medication. We did not find evidence for a relationship between medication status and spindle rate among subjects with resolved epilepsy $(p=0.18)$. We conclude that an inverse relationship exists between spindle and spike rate; the higher the spindle rate, the lower the spike rate. These results are consistent with the hypothesis that competitively shared thalamocortical circuitry supports both spikes and spindles during NREM sleep.

Spindle rate, but not spike rate, predicts cognitive function While children with CECTS are known to have deficits in cognitive function, whether the deficits in spindle rate observed here correlate with neuropsychological performance is unknown. To test this, we compare each subject's spindle rate in the centrotemporal channels with performance on four neuropsychological tests in domains commonly affecting children with CECTS: general intelligence (measured by FSIQ), fine motor dexterity (measured by the GPT), processing speed (measured by the PSI), and phonological awareness (measured by the Comprehensive Test of Phonological Processing, second edition; see Neuropsychological assessment). We found a positive relationship between spindle rate in the centrotemporal regions and FSIQ (effect size $0.96,95 \%$ CI $[0.39,1.5], p=0.0025$; Fig. 6A), fine motor dexterity (effect size $1.14,95 \%$ CI [0.53, 1.75], $p=0.00047$; Fig. $6 B$ ), and processing speed (effect size $0.75,95 \%$ CI $[0.16,1.3], p=0.018$; Fig. $6 C$ ). We did not find evidence of a relationship between spindle rate and the measure of phonological processing applied here $(p=0.34$; Fig. $6 D)$.

To assess the relationship between spike rate and cognitive function, we apply two independent methods to compute spike rate in all patients with CECTS. We found no evidence of a relationship between spike rate in the centrotemporal regions and cognitive function in all cases $(p>0.12$, except for a possible negative trend between Persyst spike detections and processing speed, $p=0.06$, Fig. $7 B$; and a possible negative trend between SpikeNet spike detections and phonological processing, $p=0.072$, Fig. $7 C$ ). Restricting analysis of spindle rate to patients with CECTS for comparison (Fig. $7 A$ ), we still found a positive relationship between spindle rate in the centrotemporal regions and FSIQ (effect size 1.24, 95\% CI [0.36, 2.11], $p=0.014$ ) and fine motor dexterity (effect size 1.2, 95\% CI [0.34, 2.06], $p=0.0092$ ), and a possible trend between spindle rate and processing speed (effect size $0.71,95 \%$ CI $[-0.11,1.53], p=0.10$ ). We conclude that relationships exist between spindle rate in the centrotemporal regions and performance on three neuropsychological tasks in CECTS; increased spindle rate correlates with improved task performance. In contrast, using two different spike detection methods, we find spike rate does not reliably correlate with neuropsychological performance.

\section{Spindle duration, amplitude, and frequency, remain consistent across groups}

To investigate the spindle characteristics of each group, we computed three features: spindle duration, frequency, and amplitude. We found no evidence that children with CECTS have altered spindle duration compared with control subjects (Fig. 8A; $p=0.15$ for A-CECTS, and $p=0.62$ for R-CECTS; see Analysis of spindle features). Across all subjects, we found a mean spindle duration of 0.88 s (SD 0.23 s). Similarly, we found no evidence that children with CECTS have altered amplitude during spindles compared with control subjects (Fig. $8 B ; p=0.15$ for ACECTS, and $p=0.74$ for R-CECTS). Across all subjects, we found a mean spindle amplitude $34.8 \mu \mathrm{V}$ (SD $12.3 \mu \mathrm{V})$. Finally, we found no evidence that children with CECTS have altered spindle frequency compared with control subjects (Fig. 8C; $p=0.61$ for A-CECTS, and $p=0.67$ for R-CECTS). Across all subjects, we found a mean $\sigma$ frequency of $12.6 \mathrm{~Hz}(\mathrm{SD} 1.0 \mathrm{~Hz})$. We conclude that spindles have similar durations, amplitudes, and frequencies in children with CECTS compared with control subjects.

\section{Consistent trends occur in a narrow frequency interval}

To broadly capture spindle frequencies in a pediatric age group, we analyzed spindles spanning a $9-15 \mathrm{~Hz}$ frequency range (Purcell et al., 2017). To explore whether consistent results occur in narrow frequency bands (Mölle et al., 2011), we first show the average power spectrum at the centrotemporal channels for each subject (see Spectral analysis; Fig. 9A). Most spectral peaks in the 

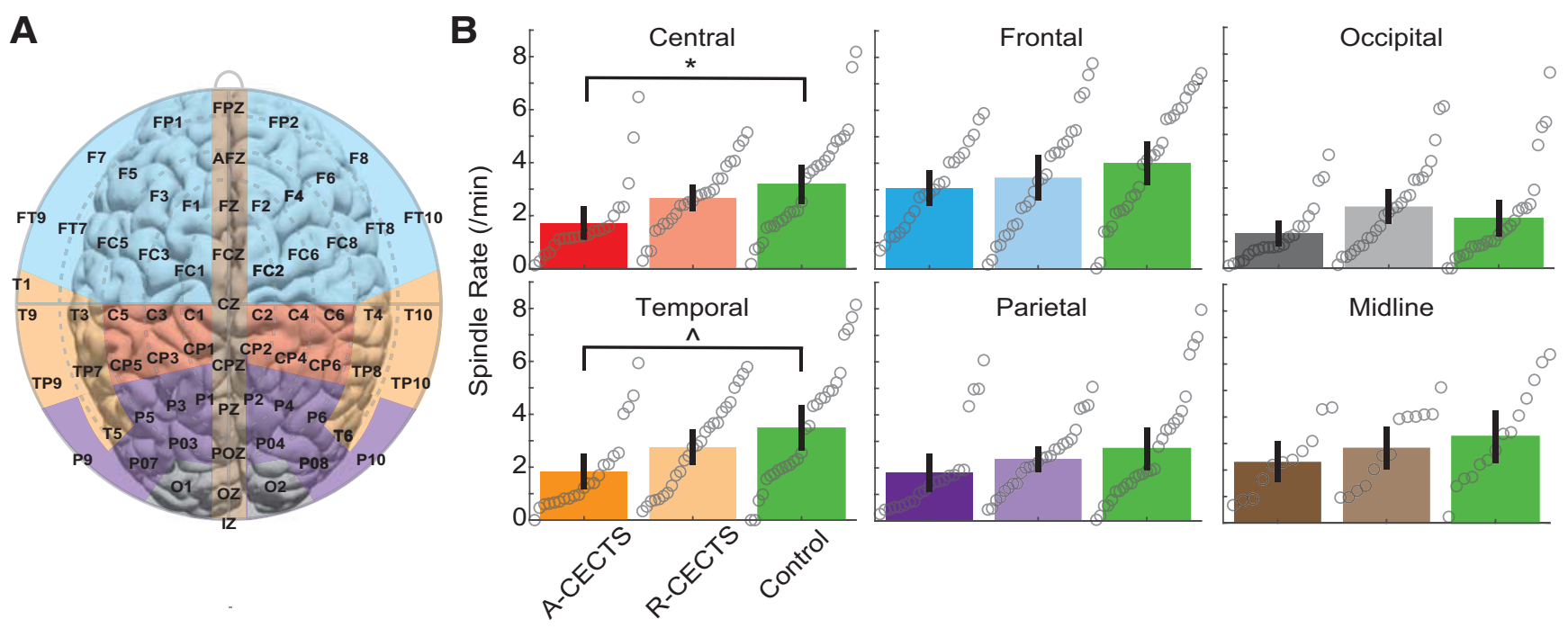

Figure 4. The reduction in spindle rate is focal. $A$, Electrode groups corresponding to: frontal (blue), central (red), parietal (purple), temporal (orange), occipital (gray), and midline (brown). $B$, Spindle rate versus subject group, as in Figure 3 , for each region. $* p=0.026 . \wedge p=0.057$.
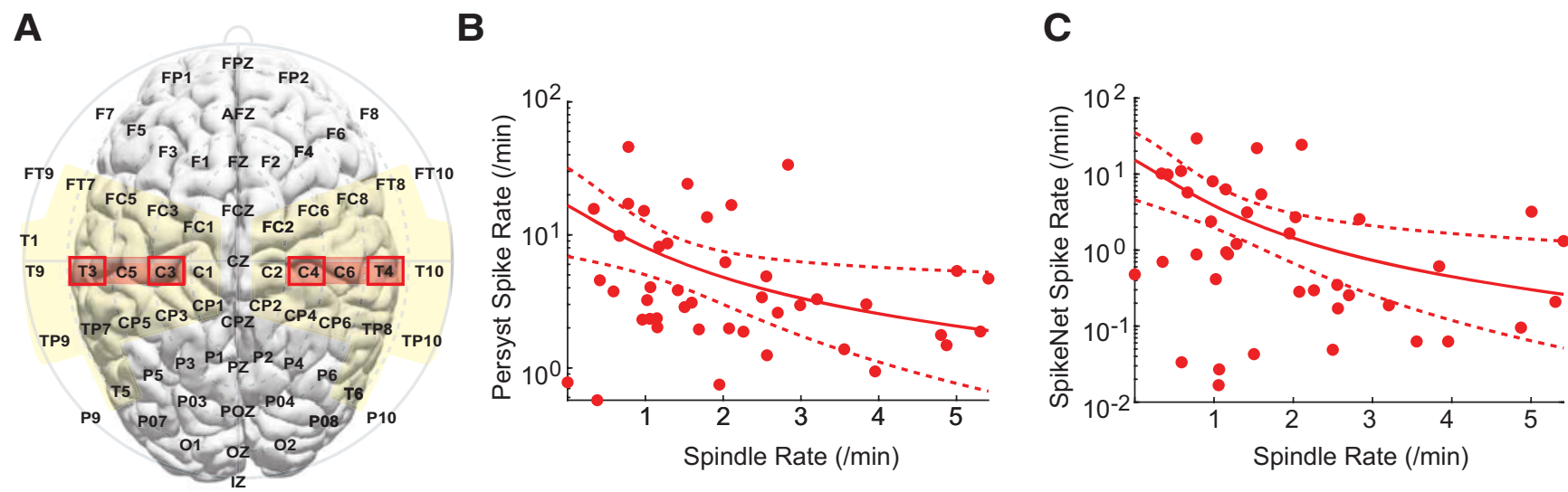

Figure 5. Spindle and spike rates are inversely related. $A$, Electrodes used to compute the average spindle rate (red regions) and the summed spike rate for two detection methods (yellow regions for Persyst, red boxes for SpikeNet, based on the montages used for these algorithms). $\boldsymbol{B}$, C, The spike rate computed with (B) Persyst and (C) SpikeNet is lower in corresponding regions with higher spindle rate. Solid curve indicates the model fit. Dashed curves indicate the $95 \% \mathrm{Cls}$.

A

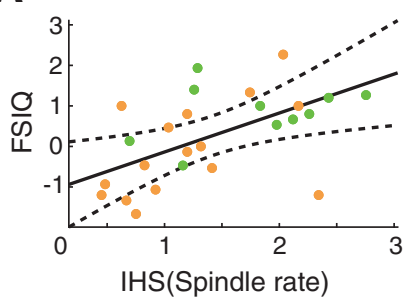

B

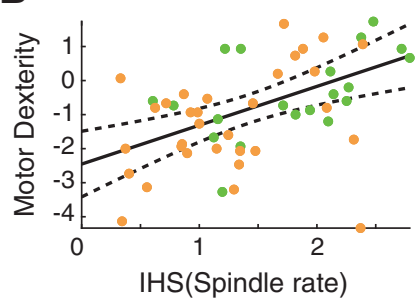

C

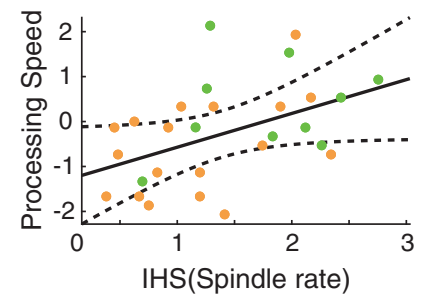

D

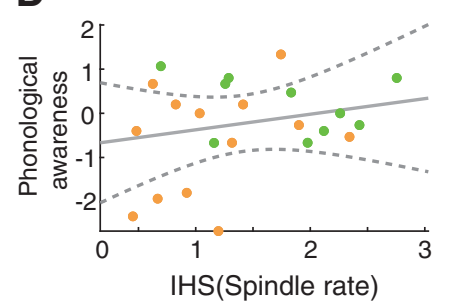

Figure 6. Spindle rate correlates with task performance. Spindle rate versus performance in four tasks: (A) full scale IQ (FSIQ), (B) motor dexterity (measured by the GPT), (C) processing speed (measured by the PSI), and (D) phonological awareness. Orange (green) circles represent CECTS (control) subjects. Black solid (dashed) lines indicate significant model fits (95\% Cls). Gray solid (dashed) lines indicate models with insignificant fits (95\% Cls).

$\sigma$ band (26 of the 27 subjects analyzed) occurred at frequencies $>12 \mathrm{~Hz}$ (Fig. 9A; mean peak frequency $13.2 \mathrm{~Hz}$, SD $0.95 \mathrm{~Hz}$ ). We therefore considered spindles detected with frequencies $9-12 \mathrm{~Hz}$ (e.g., slow spindles) and $12-15 \mathrm{~Hz}$ (e.g., fast spindles). In the 12$15 \mathrm{~Hz}$ frequency band, we found results consistent with the broadband analysis: (1) lower spindle rate in the centrotemporal regions in patients with A-CECTS $(p=0.034$; Fig. $9 B)$ compared with control subjects; (2) a possible inverse relationship between spindle and spike rates ( $p=0.040$ using SpikeNet, $p=0.15$ using Persyst; Fig. $9 C$ ); and (3) positive relationships between spindle rate and performance on three neuropsychological tests (general intelligence $p=0.013$, fine motor dexterity $p=0.0029$, and processing speed $p=0.027$; Fig. $9 D$ ). We also found evidence of focal spindle deficits in the central 


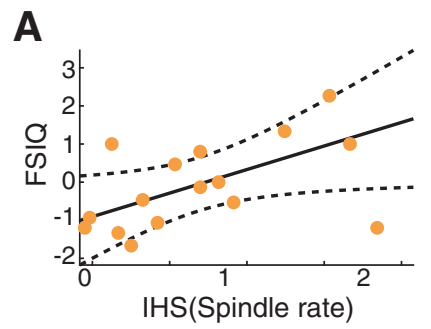

B

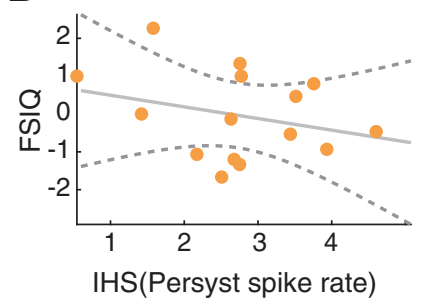

C

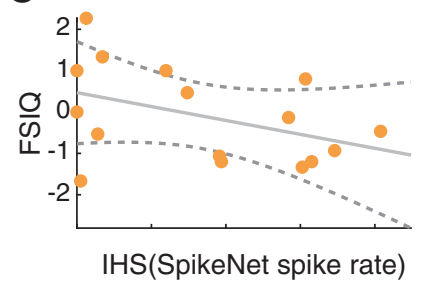

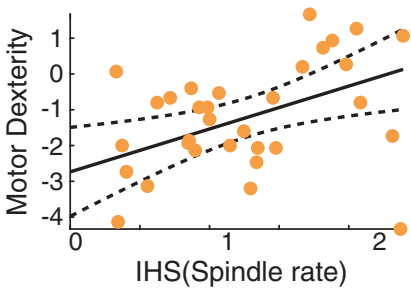
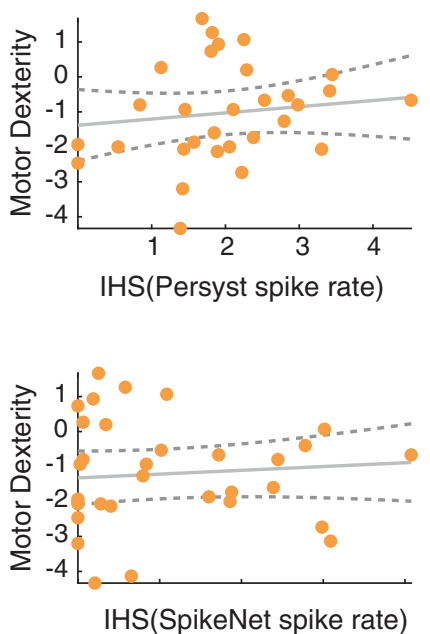
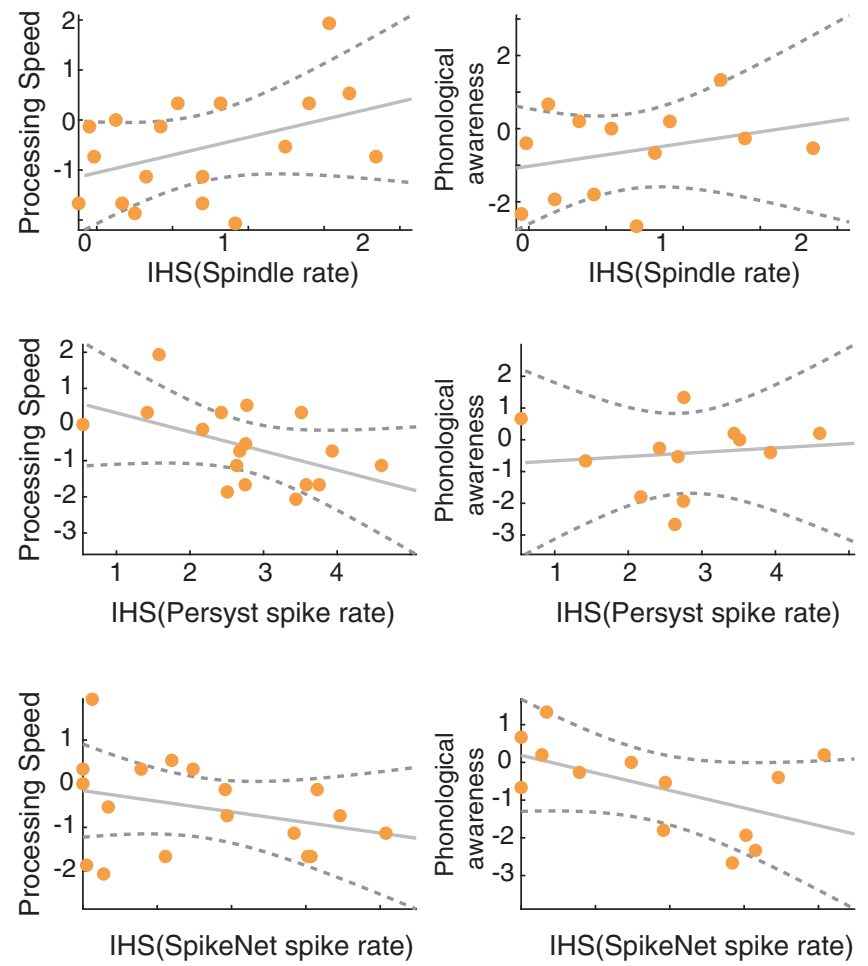

Figure 7. Spindle rate, but not spike rate, correlates with task performance. $A$, Spindle rate for only subjects with CECTS (orange circles) versus performance in four tasks: FSIQ, motor dexterity (measured by the GPT), processing speed (measured by the PSI), and phonological awareness. $\boldsymbol{B}, \boldsymbol{C}$, Spike rate computed using (B) Persyst or (C) SpikeNet versus performance in four tasks for only subjects with CECTS. Black solid (dashed) lines indicate significant model fits (95\% Cls). Gray solid (dashed) lines indicate models with insignificant fits $(95 \%$ Cls).

$(p=0.053)$ and temporal $(p=0.031)$ regions; $p>0.12$ in all other regions. For the $9-12 \mathrm{~Hz}$ frequency band, we found no evidence of a difference in spindle rates between A-CECTS and control subjects ( $p>0.22$ in all regions; Fig. $9 E)$. However, we did observe a possible inverse relationship between spindle rate and spike rate $(p=0.026$ using SpikeNet, $p=0.10$ using Persyst; Fig. $9 F$ ), and possible relationships between spindle rate and performance on neuropsychological tests (general intelligence $p=0.081$, fine motor dexterity $p=0.061$, processing speed $p=0.094$; Fig. 9G). We conclude that, while a broadband analysis captures the strongest effects, narrowband analysis in the $12-15 \mathrm{~Hz}$ range produces qualitatively similar results.

\section{Discussion}

Although CECTS is a common developmental epilepsy, the pathophysiological mechanism leading to seizures and cognitive dysfunction in these children is unknown. Here, we show that focal spindle deficits are an electrophysiological feature of CECTS. We further show that the spindle deficits are present in the active, but not resolved, disease state and correlate with the neuropsychological deficits observed. These observations have several implications for our understanding of the role of the thalamocortical circuit in the pathophysiology of this disease, the role of abnormal spindles on cognitive dysfunction in CECTS, and novel opportunities for treatment. In addition, because children with
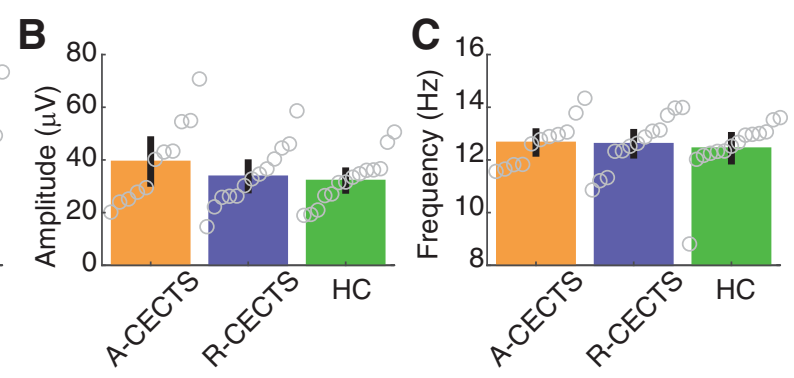

Figure 8. Spindle features are consistent across groups. The $(\boldsymbol{A})$ duration, $(\boldsymbol{B})$ amplitude, and $(\boldsymbol{C})$ frequency of detected spindles for each subject group. Circles represent values for each patient. Bar heights indicate the mean feature value. Vertical black lines indicate 2 SEMs.

CECTS overlap clinically and genetically with more severe epileptic encephalopathies (Lemke et al., 2013; Gao et al., 2017; Kessi et al., 2018; Strehlow et al., 2019), these findings may have broader applicability to these rare electroclinical epilepsy syndromes.

Prior studies have demonstrated subtle structural abnormalities involving the thalamus (Sánchez Fernández et al., 2012, 2017; Leal et al., 2018) and thalamocortical white matter in children with CECTS and related epileptic encephalopathies (Ciumas et al., 2014; S. E. Kim et al., 2014; Xiao et al., 2014; Ostrowski et al., 2019). Our finding of spindle dysfunction in CECTS also implicates disruption to the thalamocortical circuit in this disease. Spindles are generated in the reticular nucleus of the thalamus, a diffuse web-like collection of GABAergic neurons, which encase the other thalamic nuclei (Fuentealba and Steriade, 2005; Fogerson and Huguenard, 2016). These neurons project primarily to glutamatergic thalamocortical neurons, which entrain cortical areas to their $\sigma$ frequency rhythms (Beenhakker and Huguenard, 2009). 
A
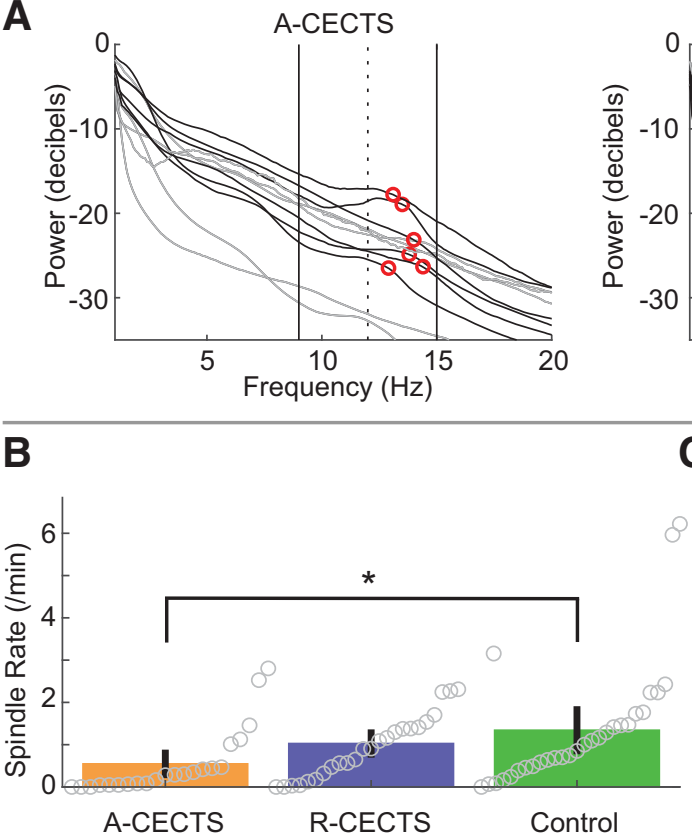

D
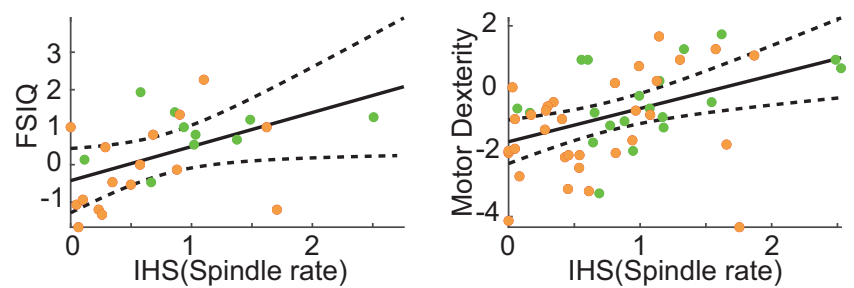
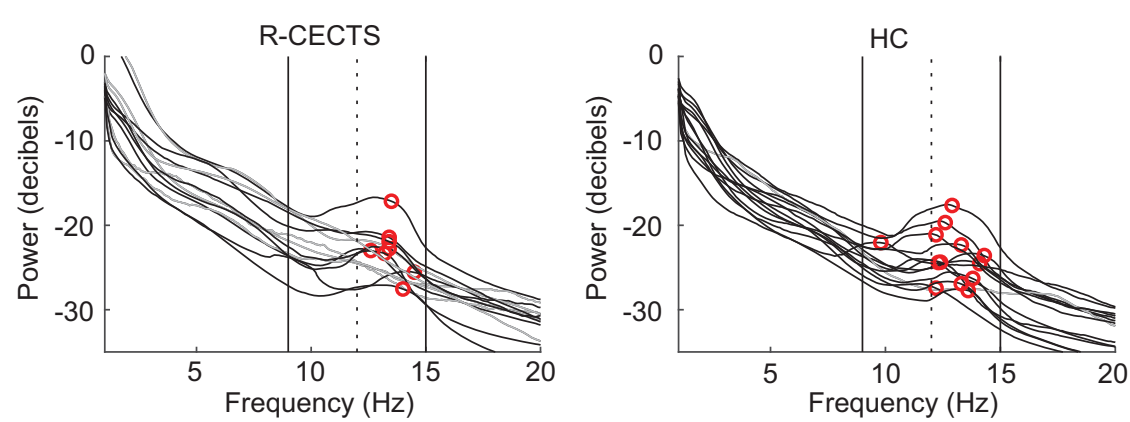

12-15 Hz
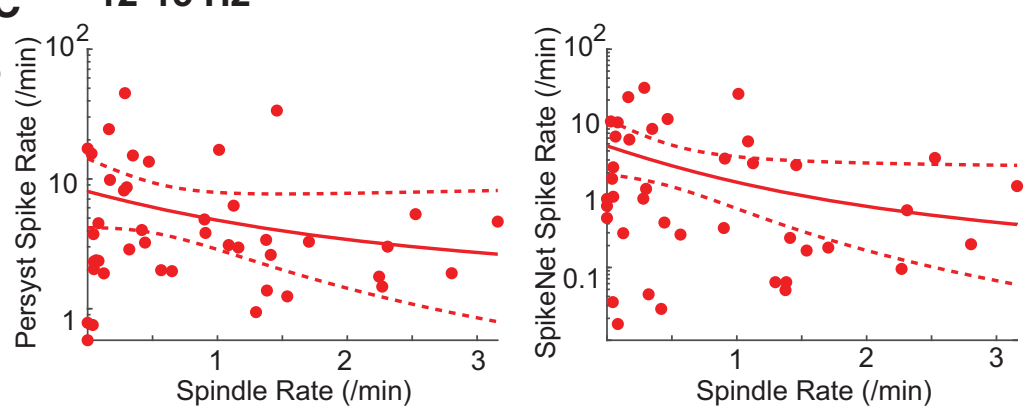
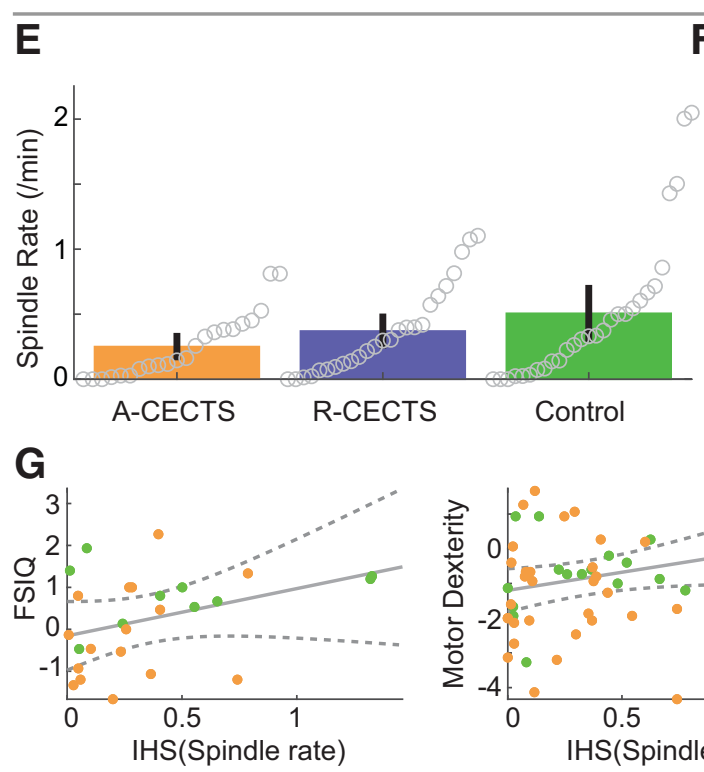

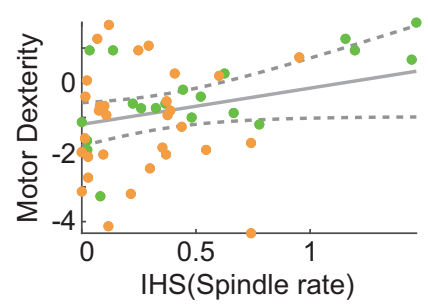

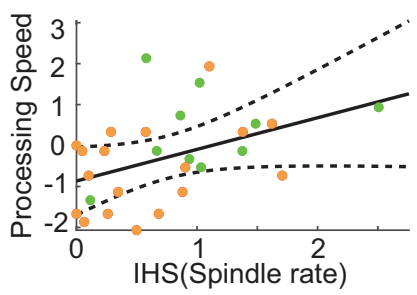

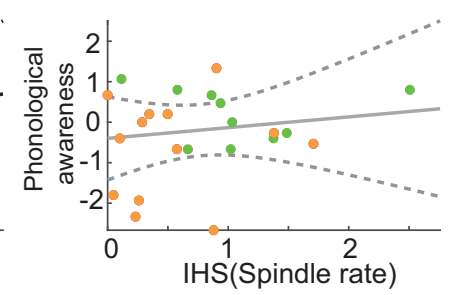

Figure 9. Analysis of fast spindles reveals consistent results. $A$, Power spectra for each subject, in each group. For most subjects, peak power in the $\sigma$ band (red circles) occurs at a frequency $>12 \mathrm{~Hz}$ (vertical dashed line). In each figure, black (gray) curves indicate subjects with (without) a local maximum in the $\sigma$ band (9-15 Hz, vertical black lines). $\boldsymbol{B}-\boldsymbol{G}$, Results of narrowband

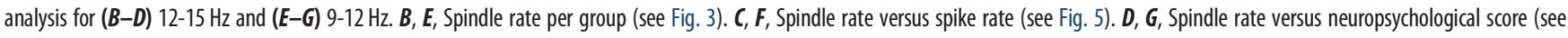
Fig. 6). $* p=0.034$

The resulting rhythms (sleep spindles) are amplified and propagated through corticocortical and thalamocortical circuits. Corticothalamic neurons send glutamatergic inputs back to the thalamus, producing a feedback loop regulated primarily by GABAergic and glutamatergic neurotransmission (Jacobsen et al., 2001).
Our observation of an inverse relationship between spike and spindle rate during NREM sleep further supports observations that spikes and spindles may represent competitive processes that use the same thalamocortical circuitry (Beenhakker and Huguenard, 2009). In vitro, both spikes and spindles are 
recorded from single thalamic cells and thalamic slice preparations after different pharmacologic treatments (von Krosigk et al., 1993; Huguenard and Prince, 1994; U. Kim et al., 1997). In humans, thalamic and cortical activity measured with depth electrodes is phase-locked during both spindles and spikes (Williams, 1953; Andersen et al., 1967). Although thalamocortical circuit dysfunction has previously been implicated in the pathophysiology of generalized seizures in absence epilepsy (Williams, 1953; Beenhakker and Huguenard, 2009; Maheshwari and Noebels, 2014; Fogerson and Huguenard, 2016), our observation of a focal spindle deficit in CECTS reveals that thalamocortical circuit can be focally disrupted in epilepsy. Consistent with this, prior work has demonstrated that distinct thalamocortical assemblages generate focal spindles (Andersen et al., 1967; Bastuji et al., 2020), and these distinct circuits can promote focal abnormalities (Fogerson and Huguenard, 2016; Clemente-Perez et al., 2017). In particular, sensorimotor thalamocortical circuits promote spatially distinct spindle populations (Nishida and Walker, 2007; Fogerson and Huguenard, 2016), and these sensorimotor spindles have been mechanistically tied to focal seizures in mouse models (Clemente-Perez et al., 2017). In a focal epilepsy rodent model, the thalamus was found to maintain cortical seizures and provide a neuromodulatory target to disrupt them (Paz et al., 2013). Our findings of disruptions specific to the centrotemporal regions in CECTS implicate either discrete thalamic nuclei (e.g., ventroanterior, ventrolateral, and ventroposterior nuclei) (Andersen et al., 1967) or discrete cell types (e.g., parvalbumin expressing neurons that are mediolaterally distributed in the reticular nucleus of the thalamus) (Clemente-Perez et al., 2017) in this disease.

Sensorimotor sleep spindles typically increase in frequency and rate over childhood (Purcell et al., 2017; Hahn et al., 2019), coincident with the age period that CECTS presents. The relationship between spindle rate and sleep-dependent consolidation of both procedural (Walker et al., 2002; Fogel and Smith, 2006; Nishida and Walker, 2007; Peters et al., 2008; Tamaki et al., 2008; Rasch et al., 2009) and declarative (Clemens et al., 2005, 2006; Schabus et al., 2008) memory is well established in healthy adults, and spindle dysfunction has been implicated as a mechanism for poor memory in aging (Pace-Schott and Spencer, 2015) and disease (Baran et al., 2018). Prior work has also demonstrated that general cognitive abilities and sleep-dependent memory consolidation are associated with spindle rate in healthy neurotypical children and those with autism (Wilhelm et al., 2012; Farmer et al., 2018; Hahn et al., 2019). Here, we find that spindles are disrupted in children with a sleep-activated focal developmental epilepsy syndrome, CECTS, and correlate with general intelligence, fine motor dexterity, and processing speed. We did not find a relationship between spindle rate and phonological processing, which may reflect an alternate mechanism behind the language challenges in these children or the limitations of our approach. Given the short samples of sleep available, we included both Stage 2 and 3 NREM sleep states in our analysis, although the rate of spindles in memory functions may differ between these states (Cox et al., 2012, 2017). In addition, we measured neuropsychological performance on a separate day from the EEG recordings. Future work, pairing spindle measures with same-day cognitive testing and including a measure of memory encoding, may provide a more sensitive assay of the dynamic relationship between spindles and cognitive function in these children. In addition, using a cross-sectional dataset of children at different stages of the disease, we found evidence for a transient spindle deficit. Confirmation that resolution of the spindle deficit coincides with resolving neuropsychological concerns will require a longitudinal study.

Children with CECTS develop a range of mild to profound cognitive deficits concurrent with seizures (Tovia et al., 2011; Ross et al., 2020). The severe epileptic encephalopathies that present over the same ages include continuous spike and wave of sleep with encephalopathy and Landau Kleffner syndrome. These syndromes are characterized by interictal spikes in $>85 \%$ of NREM sleep and permanent and progressive declines in cognitive function (Scheltens-de Boer, 2009). Supporting the phenotypic overlap between CECTS and these disorders, genetic studies reveal that the same pathologic mutation can lead to continuous spike and wave of sleep with encephalopathy or CECTS (Lemke et al., 2013; Gao et al., 2017; Kessi et al., 2018; Strehlow et al., 2019). In addition, a prospective study reported that $6.6 \%$ of CECTS cases meet diagnostic criteria for continuous spike and wave of sleep with encephalopathy or Landau Kleffner syndrome (Tovia et al., 2011). Thus, our findings of a spindle deficit and thalamocortical circuit dysfunction in CECTS may provide new insights for these related but more severe epileptic encephalopathies.

Importantly, there are currently no available validated biomarkers to identify cognitive risk or proven strategies to treat cognitive dysfunction in children with CECTS or related epileptic encephalopathies. The development and validation of a robust spindle detector for use in patients with epileptiform spikes, and the identification of a spindle deficit in CECTS, open new opportunities in both of these areas. Using a crosssectional, case-control study design, we identified spindle deficits in children with active epilepsy and a correlation between spindle measures and cognitive function. Future work, using the LS or comparably performing detector, to identify normative spindle rate values in healthy children across ages may enable efficient identification of children with epilepsy who are at risk of cognitive deficits. Further, longitudinal assessments within children with CECTS may identify those at risk of cognitive regression, or conversely, those entering resolution of cognitive disease in CECTS. In addition, given the direct role that spindles play in memory consolidation, spindle deficits offer a novel treatment target (Manoach et al., 2020). Prior work has demonstrated that interventions that increase spindle activity result in improved sleep-dependent memory consolidation (Kaestner et al., 2013; Mednick et al., 2013; Lustenberger et al., 2016; Leminen et al., 2017). Future work is required to explore the potential of spindle-promoting interventions to improve cognitive function in children with CECTS.

Evidence of spindle disruption in CECTS demonstrates thalamocortical circuit dysfunction in this idiopathic focal epilepsy and provides a common pathophysiology for the shared seizures and cognitive symptoms observed. Further, sleep spindles offer a mechanistic biomarker, and potential treatment target, of cognitive dysfunction in this common epileptic encephalopathy.

\section{References}

Andersen P, Andersson SA, Lomo T (1967) Nature of thalamo-cortical relations during spontaneous barbiturate spindle activity. J Physiol 192:283307.

Astradsson A, Olafsson E, Ludvigsson P, Bjorgvinsson H, Hauser WA (1998) Rolandic epilepsy: an incidence study in Iceland. Epilepsia 39:884-886.

Baran B, Correll D, Vuper TC, Morgan A, Durrant SJ, Manoach DS, Stickgold R (2018) Spared and impaired sleep-dependent memory consolidation in schizophrenia. Schizophr Res 199:83-89. 
Bastuji H, Lamouroux P, Villalba M, Magnin M, Garcia-Larrea L (2020) Local sleep spindles in the human thalamus. J Physiol 598:2109-2124.

Beenhakker MP, Huguenard JR (2009) Neurons that fire together also conspire together: is normal sleep circuitry hijacked to generate epilepsy? Neuron 62:612-632.

Benjamini Y, Hochberg Y (1995) Controlling the false discovery rate: a practical and powerful approach to multiple testing. J R Stat Soc B57:289-300.

Berg AT, Rychlik K (2015) The course of childhood-onset epilepsy over the first two decades: a prospective, longitudinal study. Epilepsia 56:40-48.

Berg AT, Berkovic SF, Brodie MJ, Buchhalter J, Cross JH, van Emde Boas W, Engel J, French J, Glauser TA, Mathern GW, Moshé SL, Nordli D, Plouin P, Scheffer IE (2010) Revised terminology and concepts for organization of seizures and epilepsies: report of the ILAE Commission on Classification and Terminology, 2005-2009. Epilepsia 51:676-685.

Bjørnæs H, Bakke KA, Larsson PG, Heminghyt E, Rytter E, Brager-Larsen LM, Eriksson AS (2013) Subclinical epileptiform activity in children with electrical status epilepticus during sleep: effects on cognition and behavior before and after treatment with levetiracetam. Epilepsy Behav 27:4048.

Bouma PA, Bovenkerk AC, Westendorp RG, Brouwer OF (1997) The course of benign partial epilepsy of childhood with centrotemporal spikes: a meta-analysis. Neurology 48:430-437.

Burbidge JB, Magee L, Robb AL (1988) Alternative transformations to handle extreme values of the dependent variable. J Am Stat Assoc 83:123-127.

Callenbach PM, Bouma PA, Geerts AT, Arts WF, Stroink H, Peeters EA, Donselaar CA, van Peters AC, Brouwer OF (2010) Long term outcome of benign childhood epilepsy with centrotemporal spikes: Dutch Study of Epilepsy in Childhood. Seizure 19:501-506.

Camfield CS, Camfield PR (2014) Rolandic epilepsy has little effect on adult life 30 years later. Neurology 82:1162-1166.

Carvill GL, Regan BM, Yendle SC, O'Roak BJ, Lozovaya N, Bruneau N, Burnashev N, Khan A, Cook J, Geraghty E, Sadleir LG, Turner SJ, Tsai MH, Webster R, Ouvrier R, Damiano JA, Berkovic SF, Shendure J, Hildebrand MS, Szepetowski P, et al. (2013) GRIN2A mutations cause epilepsy-aphasia spectrum disorders. Nat Genet 45:1073-1076.

Ciumas C, Saignavongs M, Ilski F, Herbillon V, Laurent A, Lothe A, Heckemann RA, de Bellescize J, Panagiotakaki E, Hannoun S, Marinier DS, Montavont A, Ostrowsky-Coste K, Bedoin N, Ryvlin P (2014) White matter development in children with benign childhood epilepsy with centro-temporal spikes. Brain 137:1095-1106.

Clemens Z, Fabó D, Halász P (2005) Overnight verbal memory retention correlates with the number of sleep spindles. Neuroscience 132:529-535.

Clemens Z, Fabó D, Halász P (2006) Twenty-four hours retention of visuospatial memory correlates with the number of parietal sleep spindles. Neurosci Lett 403:52-56.

Clemente-Perez A, Makinson SR, Higashikubo B, Brovarney S, Cho FS, Urry A, Holden SS, Wimer M, Dávid C, Fenno LE, Acsády L, Deisseroth K, Paz JT (2017) Distinct thalamic reticular cell types differentially modulate normal and pathological cortical rhythms. Cell Rep 19:2130-2142.

Cox R, Hofman WF, Talamini LM (2012) Involvement of spindles in memory consolidation is slow wave sleep-specific. Learn Mem 19:264-267.

Cox R, Schapiro AC, Manoach DS, Stickgold R (2017) Individual differences in frequency and topography of slow and fast sleep spindles. Front Hum Neurosci 11:433.

Dreifuss F, Martinez-Lage M, Roger J, Seino M, Wolf P, Dam M (1989) Proposal for revised classification of epilepsies and epileptic syndromes. Epilepsia 30:389-399.

Eden UT, Kramer MA (2010) Drawing inferences from Fano factor calculations. J Neurosci Methods 190:149-152.

Farmer CA, Chilakamarri P, Thurm AE, Swedo SE, Holmes GL, Buckley AW (2018) Spindle activity in young children with autism, developmental delay, or typical development. Neurology 91:e112-e122.

Fisher RS, Acevedo C, Arzimanoglou A, Bogacz A, Cross JH, Elger CE, Engel J, Forsgren L, French JA, Glynn M, Hesdorffer DC, Lee BI, Mathern GW, Moshé SL, Perucca E, Scheffer IE, Tomson T, Watanabe M, Wiebe S (2014) ILAE official report: a practical clinical definition of epilepsy. Epilepsia 55:475-482.
Fogel SM, Smith CT (2006) Learning-dependent changes in sleep spindles and Stage 2 sleep. J Sleep Res 15:250-255.

Fogerson PM, Huguenard JR (2016) Tapping the brakes: cellular and synaptic mechanisms that regulate thalamic oscillations. Neuron 92:687704.

Fuentealba P, Steriade M (2005) The reticular nucleus revisited: intrinsic and network properties of a thalamic pacemaker. Prog Neurobiol 75:125-141.

Gao K, Tankovic A, Zhang Y, Kusumoto H, Zhang J, Chen W, XiangWei W, Shaulsky GH, Hu C, Traynelis SF, Yuan H, Jiang Y (2017) A de novo loss-of-function GRIN2A mutation associated with childhood focal epilepsy and acquired epileptic aphasia. PLoS One 12:e170818.

Gelman A (2005) Analysis of variance: why it is more important than ever. Ann Statist 33:1-53.

Gent TC, Bassetti CL, Adamantidis AR (2018) Sleep-wake control and the thalamus. Curr Opin Neurobiol 52:188-197.

Grigg-Damberger M, Gozal D, Marcus CL, Quan SF, Rosen CL, Chervin RD, Wise M, Picchietti DL, Sheldon SH, Iber C (2007) The visual scoring of sleep and arousal in infants and children. J Clin Sleep Med 03:201-240

Hahn M, Joechner AK, Roell J, Schabus M, Heib DP, Gruber G, Peigneux P, Hoedlmoser K (2019) Developmental changes of sleep spindles and their impact on sleep-dependent memory consolidation and general cognitive abilities: a longitudinal approach. Dev Sci 22:e12706.

Helfrich RF, Lendner JD, Mander BA, Guillen H, Paff M, Mnatsakanyan L, Vadera S, Walker MP, Lin JJ, Knight RT (2019) Bidirectional prefrontalhippocampal dynamics organize information transfer during sleep in humans. Nat Commun 10:16

Huguenard JR, Prince DA (1994) Intrathalamic rhythmicity studied in vitro: nominal T-current modulation causes robust antioscillatory effects. J Neurosci 14:5485-5502.

Iber C, Ancoli-Israel S, Chesson A, Quan S (2007) AASM manual for the scoring of sleep and associated events. Darien, IL: American Academy of Sleep Medicine.

Jacobsen RB, Ulrich D, Huguenard JR (2001) GABA(B) and NMDA receptors contribute to spindle-like oscillations in rat thalamus in vitro. J Neurophysiol 86:1365-1375.

Jacobson LA, Ryan M, Martin RB, Ewen J, Mostofsky SH, Denckla MB, Mahone EM (2011) Working memory influences processing speed and reading fluency in ADHD. Child Neuropsychol 17:209-224.

Jing J, Sun H, Kim JA, Herlopian A, Karakis I, Ng M, Halford JJ, Maus D, Chan F, Dolatshahi M, Muniz C, Chu C, Sacca V, Pathmanathan J, Ge W, Dauwels J, Lam A, Cole AJ, Cash SS, Westover MB (2020a) Development of expert-level automated detection of epileptiform discharges during electroencephalogram interpretation. JAMA Neurol 77:103-108.

Jing J, Herlopian A, Karakis I, Ng M, Halford JJ, Lam A, Maus D, Chan F, Dolatshahi M, Muniz CF, Chu C, Sacca V, Pathmanathan J, Ge W, Sun H, Dauwels J, Cole AJ, Hoch DB, Cash SS, Westover MB (2020b) Interrater reliability of experts in identifying interictal epileptiform discharges in electroencephalograms. JAMA Neurol 77:49-57.

Joshi CN, Chapman KE, Bear JJ, Wilson SB, Walleigh DJ, Scheuer ML (2018) Semiautomated spike detection software Persyst 13 is noninferior to human readers when calculating the spike-wave index in electrical status epilepticus in sleep. J Clin Neurophysiol 35:370-374.

Kaestner EJ, Wixted JT, Mednick SC (2013) Pharmacologically increasing sleep spindles enhances recognition for negative and high-arousal memories. J Cogn Neurosci 25:1597-1610.

Kessi M, Peng J, Yang L, Xiong J, Duan H, Pang N, Yin F (2018) Genetic etiologies of the electrical status epilepticus during slow wave sleep: systematic review. BMC Genet 19:40.

Kim SE, Lee JH, Chung HK, Lim SM, Lee HW (2014) Alterations in white matter microstructures and cognitive dysfunctions in benign childhood epilepsy with centrotemporal spikes. Eur J Neurol 21:708-717.

Kim U, Sanchez-Vives MV, McCormick DA (1997) Functional dynamics of GABAergic inhibition in the thalamus. Science 278:130134.

Kramer MA, Tort AB, Kopell NJ (2008) Sharp edge artifacts and spurious coupling in EEG frequency comodulation measures. J Neurosci Methods 170:352-357. 
Kramer MA, Ostrowski LM, Song DY, Thorn EL, Stoyell SM, Parnes M, Chinappen D, Xiao G, Eden UT, Staley KJ, Stufflebeam SM, Chu CJ (2019) Scalp recorded spike ripples predict seizure risk in childhood epilepsy better than spikes. Brain 142:1296-1309.

Larsson K, Eeg-Olofsson O (2006) A population based study of epilepsy in children from a Swedish county. Eur J Paediatr Neurol 10:107-113.

Leal A, Calado E, Vieira JP, Mendonça C, Ferreira JC, Ferreira H, Carvalho D, Furtado F, Gomes R, Monteiro JP (2018) Anatomical and physiological basis of continuous spike-wave of sleep syndrome after early thalamic lesions. Epilepsy Behav 78:243-255.

Leminen MM, Virkkala J, Saure E, Paajanen T, Zee PC, Santostasi G, Hublin C, Müller K, Porkka-Heiskanen T, Huotilainen M, Paunio T (2017) Enhanced memory consolidation via automatic sound stimulation during non-REM sleep. Sleep 40:zsx003.

Lemke JR, Lal D, Reinthaler EM, Steiner I, Nothnagel M, Alber M, Geider K, Laube B, Schwake M, Finsterwalder K, Franke A, Schilhabel M, Jähn JA, Muhle H, Boor R, Van Paesschen W, Caraballo R, Fejerman N, Weckhuysen S, De Jonghe P, et al. (2013) Mutations in GRIN2A cause idiopathic focal epilepsy with Rolandic spikes. Nat Genet 45:1067-1072.

Lustenberger C, Boyle MR, Alagapan S, Mellin JM, Vaughn BV, Fröhlich F (2016) Feedback-controlled transcranial alternating current stimulation reveals a functional role of sleep spindles in motor memory consolidation. Curr Biol 26:2127-2136.

Maheshwari A, Noebels JL (2014) Monogenic models of absence epilepsy: windows into the complex balance between inhibition and excitation in thalamocortical microcircuits. Prog Brain Res 213: 223-252.

Manoach DS, Mylonas D, Baxter B (2020) Targeting sleep oscillations to improve memory in schizophrenia. Schizophr Res 221:63-70.

McCormick DA, Bal T (1997) Sleep and arousal: thalamocortical mechanisms. Annu Rev Neurosci 20:185-215.

Mednick SC, McDevitt EA, Walsh JK, Wamsley E, Paulus M, Kanady JC, Drummond SPA (2013) The critical role of sleep spindles in hippocampal-dependent memory: a pharmacology study. J Neurosci 33:44944504.

Merker B, Podell K (2011) Grooved Pegboard Test. In: Encyclopedia of clinical neuropsychology (Kreutzer JS, DeLuca J, Caplan B, eds), pp 11761178. New York: Springer.

Mölle M, Bergmann TO, Marshall L, Born J (2011) Fast and slow spindles during the sleep slow oscillation: disparate coalescence and engagement in memory processing. Sleep 34:1411-1421.

Niethard N, Ngo HV, Ehrlich I, Born J (2018) Cortical circuit activity underlying sleep slow oscillations and spindles. Proc Natl Acad Sci USA 115: E9220-E9229.

Nishida M, Walker MP (2007) Daytime naps, motor memory consolidation and regionally specific sleep spindles. PLoS One 2:e341.

Nunez P, Srinivasan R (2005) Electric fields of the brain. Oxford: Oxford UP.

Ostrowski LM, Song DY, Thorn EL, Ross EE, Stoyell SM, Chinappen DM, Eden UT, Kramer MA, Emerton BC, Morgan AK, Stufflebeam SM, Chu CJ (2019) Dysmature superficial white matter microstructure in developmental focal epilepsy. Brain Commun 1:fcz002.

Pace-Schott EF, Spencer RM (2015) Sleep-dependent memory consolidation in healthy aging and mild cognitive impairment. In: Sleep, neuronal plasticity and brain function (Meerlo P, BencaRM, Abel T, eds), pp 307-330. Berlin: Springer.

Paz JT, Davidson TJ, Frechette ES, Delord B, Parada I, Peng K, Deisseroth K, Huguenard JR (2013) Closed-loop optogenetic control of thalamus as a tool for interrupting seizures after cortical injury. Nat Neurosci 16:64-70.

Peters KR, Ray L, Smith V, Smith C (2008) Changes in the density of stage 2 sleep spindles following motor learning in young and older adults. J Sleep Res 17:23-33.

Pinto AL, Fernández IS, Peters JM, Manganaro S, Singer JM, Vendrame M, Prabhu SP, Loddenkemper T, Kothare SV (2014) Localization of sleep spindles, k-complexes, and vertex waves with subdural electrodes in children. J Clin Neurophysiol 31:367-374.

Purcell SM, Manoach DS, Demanuele C, Cade BE, Mariani S, Cox R, Panagiotaropoulou G, Saxena R, Pan JQ, Smoller JW, Redline S, Stickgold R (2017) Characterizing sleep spindles in 11,630 individuals from the National Sleep Research Resource. Nat Commun $8: 15930$.
Rasch B, Pommer J, Diekelmann S, Born J (2009) Pharmacological REM sleep suppression paradoxically improves rather than impairs skill memory. Nat Neurosci 12:396-397.

Rosanova M, Ulrich D (2005) Pattern-specific associative long-term potentiation induced by a sleep spindle-related spike train. J Neurosci 25:9398-9405.

Ross EE, Stoyell SM, Kramer MA, Berg AT, Chu CJ (2020) The natural history of seizures and neuropsychiatric symptoms in BECTS: results from a large prospective, longitudinal US cohort. Epilepsy Behav 103(Pt A): 106437.

Sánchez Fernández I, Takeoka M, Tas E, Peters JM, Prabhu SP, Stannard KM, Gregas M, Eksioglu Y, Rotenberg A, Riviello JJ, Kothare SV, Loddenkemper T (2012) Early thalamic lesions in patients with sleep-potentiated epileptiform activity. Neurology 78:1721-1727.

Sánchez Fernández I, Peters JM, An S, Bergin AM, Takeoka M, Rotenberg A, Kothare SV, Riviello JJ, Loddenkemper T (2013) Long-term response to high-dose diazepam treatment in continuous spikes and waves during sleep. Pediatr Neurol 49:163-170.e4.

Sánchez Fernández I, Peters JM, Akhondi-Asl A, Klehm J, Warfield SK, Loddenkemper T (2017) Reduced thalamic volume in patients with electrical status epilepticus in sleep. Epilepsy Res 130:74-80.

Schabus M, Hoedlmoser K, Pecherstorfer T, Anderer P, Gruber G, Parapatics S, Sauter C, Kloesch G, Klimesch W, Saletu B, Zeitlhofer J (2008) Interindividual sleep spindle differences and their relation to learningrelated enhancements. Brain Res 1191:127-135.

Scheffer IE, Berkovic S, Capovilla G, Connolly MB, French J, Guilhoto L, Hirsch E, Jain S, Mathern GW, Moshé SL, Nordli DR, Perucca E, Tomson T, Wiebe S, Zhang YH, Zuberi SM (2017) ILAE classification of the epilepsies: position paper of the ILAE Commission for Classification and Terminology. Epilepsia 58:512-521.

Scheltens-de Boer M (2009) Guidelines for EEG in encephalopathy related to ESES/CSWS in children. Epilepsia 50 Suppl 7:13-17.

Scheuer ML, Bagic A, Wilson SB (2017) Spike detection: inter-reader agreement and a statistical Turing test on a large data set. Clin Neurophysiol 128:243-250.

Siapas AG, Wilson MA (1998) Coordinated interactions between hippocampal ripples and cortical spindles during slow-wave sleep. Neuron 21:1123-1128.

Sirota A, Csicsvari J, Buhl D, Buzsáki G (2003) Communication between neocortex and hippocampus during sleep in rodents. Proc Natl Acad Sci USA 100:2065-2069.

Steriade M (2005) Sleep, epilepsy and thalamic reticular inhibitory neurons. Trends Neurosci 28:317-324.

Strehlow V, Heyne HO, Vlaskamp DR, Marwick KF, Rudolf G, de Bellescize J, Biskup S, Brilstra EH, Brouwer OF, Callenbach PM, Hentschel J, Hirsch E, Kind PC, Mignot C, Platzer K, Rump P, Skehel PA, Wyllie DJ, Hardingham GE, van Ravenswaaij-Arts CM, et al. (2019) GRIN2Arelated disorders: genotype and functional consequence predict phenotype. Brain 142:80-92.

Tamaki M, Matsuoka T, Nittono H, Hori T (2008) Fast sleep spindle (13-15 $\mathrm{Hz}$ ) activity correlates with sleep-dependent improvement in visuomotor performance. Sleep 31:204-211.

Tovia E, Goldberg-Stern H, Ben Zeev B, Heyman E, Watemberg N, FattalValevski A, Kramer U (2011) The prevalence of atypical presentations and comorbidities of benign childhood epilepsy with centrotemporal spikes. Epilepsia 52:1483-1488.

Tsai MH, Vears DF, Turner SJ, Smith RL, Berkovic SF, Sadleir LG, Scheffer IE (2013) Clinical genetic study of the epilepsy-aphasia spectrum. Epilepsia 54:280-287.

Vannest J, Tenney JR, Gelineau-Morel R, Maloney T, Glauser TA (2015) Cognitive and behavioral outcomes in benign childhood epilepsy with centrotemporal spikes. Epilepsy Behav 45:85-91.

Vega C, Sánchez Fernández I, Peters J, Thome-Souza MS, Jackson M, Takeoka M, Wilkening GN, Pearl PL, Chapman K, Loddenkemper T (2018) Response to clobazam in continuous spike-wave during sleep. Dev Med Child Neurol 60:283-289.

von Krosigk M, Bal T, McCormick DA (1993) Cellular mechanisms of a synchronized oscillation in the thalamus. Science 261:361-364.

Wagner R, Torgesen J, Rashotte C (1991) Comprehensive Test of Phonological Processing: examiner's manual. Austin, TX: Sage.

Walker MP, Brakefield T, Morgan A, Hobson JA, Stickgold R (2002) Practice with sleep makes perfect: sleep-dependent motor skill learning. Neuron 35:205-211. 
Wamsley EJ, Tucker MA, Shinn AK, Ono KE, McKinley SK, Ely AV, Goff DC, Stickgold R, Manoach DS (2012) Reduced sleep spindles and spindle coherence in schizophrenia: mechanisms of impaired memory consolidation? Biol Psychiatry 71:154-161.

Warby SC, Wendt SL, Welinder P, Munk EG, Carrillo O, Sorensen HB, Jennum P, Peppard PE, Perona P, Mignot E (2014) Sleep-spindle detection: crowdsourcing and evaluating performance of experts, non-experts and automated methods. Nat Methods 11:385-392.

Webber WR, Litt B, Lesser RP, Fisher RS, Bankman I (1993) Automatic EEG spike detection: what should the computer imitate? Electroencephalogr Clin Neurophysiol 87:364-373.

Wechsler D (2014) Wechsler Intelligence Scale for Children (WISC-V), Ed 5. San Antonio, TX: Psychological Corporation.

Wickens S, Bowden SC, D'Souza W (2017) Cognitive functioning in children with self-limited epilepsy with centrotemporal spikes: a systematic review and meta-analysis. Epilepsia 58:1673-1685.
Wilhelm I, Metzkow-Mészàros M, Knapp S, Born J (2012) Sleep-dependent consolidation of procedural motor memories in children and adults. The pre-sleep level of performance matters: sleep-dependent consolidation of motor memories. Dev Sci 15:506-515.

Williams D (1953) A study of thalamic and cortical rhythms in petit mal. Brain 76:50-69.

Winter B (2013) Linear models and linear mixed-effects models in R with linguistic applications. ArXiv 1308.5499 [Cs].

Xiao F, Chen Q, Yu X, Tang Y, Luo C, Fang J, Liu L, Huang X, Gong Q, Zhou D (2014) Hemispheric lateralization of microstructural white matter abnormalities in children with active benign childhood epilepsy with centrotemporal spikes (BECTS): a preliminary DTI study. J Neurol Sci 336:171-179.

Xie W, Ross EE, Kramer MA, Eden UT, Chu CJ (2018) Timing matters: impact of anticonvulsant drug treatment and spikes on seizure risk in benign epilepsy with centrotemporal spikes. Epilepsia Open 3:409-417. 